\title{
Couching the brain's multiple-demand and language-specific systems within a macroscale gradient of cortical connectivity
}

Rocco Chiou $^{1,4} \square$, Elizabeth Jefferies ${ }^{2}$, John Duncan ${ }^{1,3}$, Gina F. Humphreys ${ }^{1}$, and Matthew A. Lambon Ralph ${ }^{1} \square$

${ }^{1}$ MRC Cognition \& Brain Sciences Unit, University of Cambridge, UK

${ }^{2}$ Department of Psychology, University of York, UK

${ }^{3}$ Department of Experimental Psychology, University of Oxford, UK

${ }^{4}$ Wellcome Centre for Integrative Neuroimaging, University of Oxford, UK

Correspondence to this work can be addressed to either of the authors by email:

(RC: rocco.chiou@ndcn.ox.ac.uk, MALR: Matt.Lambon-Ralph@mrc-cbu.cam.ac.uk).

Abstract: $\quad 200$ words

No. of Figure: $\quad 7$ items

Main text: $\quad 10,912$ words

ORCID: 0000-0002-3009-8075 (RC); 0000-0002-3826-4330 (EJ); 0000-0002-9695-2764 (JD); 0000-0001-5907-2488 (MALR)

Acknowledgements: This research was funded by an MRC programme grant and intramural funding to MALR (MR/R023883/1; MC_UU_00005/18), as well as a Sir Henry Wellcome Fellowship (201381/Z/16/Z) to RC.

Conflict of interest: The authors declare no competing financial interests. 


\begin{abstract}
The cerebrum comprises a set of specialised systems that tile across the cortical sheet, forming a tapestry-like configuration. For example, the multiple-demand and language-specific systems occupy largely separate neural estates and exhibit disparate functional profiles. Although delimiting the boundary between systems informs where cortical sheet functionally fractionates, it remains unclear why different systems' topographical placements are spatially configured in typical manners and how a macroscale architecture arises from this topography. Novel approaches have tackled this challenge by condensing the topography into a principal gradient, which represents the workflow of information processing from sensory-motoric to abstract-cognitive. To understand how the multiple-demand and language-specific systems are accommodated in the gradient framework, here we used fMRI to probe cognitive operations in semantic vs. visuospatial domains and projected functional activities onto the principal gradient. We found that the two systems showed distinct trajectories of distribution along gradient tiers, suggesting different roles in the transition from sensation to cognition. Critically, when semantic processing became difficult, the brain recruited a specialised 'semantic-control' system that was a functional and anatomical 'hybrid' juxtaposed between the multi-demand and language systems. We discuss how the brain's modular division can be better understood through the lens of a dimensionality-reduced gradient-like architecture.
\end{abstract}




\section{Introduction}

The scientific enterprise of human brain mapping is aimed at delineating the scopes and boundaries of brain regions, often in ways similar to creating a geographic representation of land. Analogous to a multitude of ways whereby geographic maps of a landmass can be characterised using different data (climatic, geologic, demographic, etc.), the brain can also be depicted using various features (microstructures, connectivity, functional tuning, etc.). Decades of research in brain cartography have culminated in two disparate approaches to characterise the spatial relationship between brain regions. Although the two approaches differ in the ways brain topography is configured and quantified, it is imperative to emphasise that, rather than mutually exclusive, the two approaches are complementary and can be used together to highlight different aspects of the brain's morphology. The first approach depicts the cortical sheet as a mosaic-like patchwork (e.g., Yeo et al. 2011; Glasser et al. 2016): The cortex comprises a number of functional distinct and spatially dispersed modules/networks, tiling across the cortex with a mottled layout of distinctly coloured blobs. In this scheme, brain regions coded with the same colour tend to exhibit similar physiological and computational characteristics, whereas brain areas of different colours fall into separate modules with distinct structural/functional traits. The second approach characterises the cortex with a number of 'principal components' (e.g., Margulies et al. 2016; Oligschläger et al. 2017). Rather than drawing boundary between networks, this approach applies dimensionality-reduction methods to condense topographical changes across the cortical mantle into a concise, lower-dimensional space. Each axis of the lower-dimensional space represents stepwise variation along a neurocognitive feature (akin to progression along a gradient). A brain region corresponds to a point in this space with its distinctive profile of functional and anatomical properties, while the distance between two points indicates the extent to which two brain regions diverge on their functional and anatomical profiles.

Representing the spatial arrangements of different brain modules with a mosaic-like layout certainly has a strong appeal. This approach is visually comprehensible, making the topographical information conspicuous with a colour-coded scheme, and has been widely used to delineate the placements of brain systems. Although different studies using this approach parcellated the brain with different scales of granularity and reported different numbers of systems (e.g., Power et al. 2011; Yeo et al. 2011; Glasser et al. 2016), a consistent result was that brain systems are topographically arranged in a highly reproducible fashion, replicated across various datasets and methodologies (for review, see Wig et al. 2014; Uddin et al. 2019). Intriguingly, the parcellation approach reveals a puzzling pattern regarding the conformation of brain systems - while some modules are spatially 'intact' (namely, they occupy a contiguous chunk/strip of cortex, such as the visual and sensorimotor cortex), other modules are spatially not contiguous and their constituent regions are scattered across different lobes 
(with long-range circuitry linking them). For instance, many systems involved in high-order functions, such as executive-control or language, are widely distributed. To explain why different systems differ in their extent of spatial contiguity, Margulies et al. (2016) gleaned insight from the gradual changes of functional tuning seen in sensory-motoric cortices. At a local-regional level, abrupt changes of tuning can arise at areal borders; however, at a system-level, subregions of a system often collectively constitute a systematic map with orderly arrangements of adjoining areas. This has been observed in the somatosensory mapping that represents the cortical homunculus (adjacent body parts correspond to adjacent cortical representations; Sanchez-Panchuelo et al. 2010) and the retinotopic mapping of receptive fields in the visual cortex (Wandell et al. 2007). Extending beyond the sensorimotor cortex, Margulies et al. (2016) tested whether, at a macroscale level of whole-brain connectome, there is an overarching architecture wherein the configuration of brain systems systematically varied from contiguous to fragmented. To this end, they used the Human Connectome Project's massive data and analytically reduced the complexity of connectivity patterns into multiple whole-brain gradients: The principal gradient reflected the 'depth' of information processing (from sensorimotor to cognition) and captured the largest share of variance, while the second gradient reflected the difference between sensory modalities. The principal gradient is thought to reflect hierarchical integration from the sensory-motoric cortex, through intermediate bimodality regions, to the default-mode network that shows transmodal reaction. When the canonical 7-network parcellation of resting-state connectivity (Yeo et al. 2011) was projected onto the principal gradient, Margulies et al. found an orderly mapping: Networks that comprise unimodal cortex (i.e., visual, auditory, sensorimotor), despite them having different input modalities, were all mapped to lower tiers of the gradient; this was followed by the dorsal/ventral attention network and the salience/executive network, and finally culminated in the transmodal default-mode network situated on the cortical apex. This orderly transition married the progression of information processing (from perceptible to abstract) with resting-state networks. Moreover, this gradient framework captured the hierarchical relationship amongst ostensibly separable modules. Although different networks are extracted as stand-alone units with their independent profiles of connectivity (typically, the orthogonality between networks is ascertained using signal decomposition, such as the ICA), the gradient analysis was able to identify an overarching organisational principle that explains how the necessity to integrate multiple sources of information drives a macroscale morphology in which networks are spatially configured in specific ways (Margulies et al. 2020). In this configuration, the default-mode network is not only 'figuratively' (in an information-processing sense) but also 'geodetically' (in a physical sense) maximally distant from the primary sensory-motoric regions. 
The macroscale gradient of cortical connectivity provides a unique framework for understanding how the neural substrates for different types of cognitive operation are couched within a bigger picture of the topographic connectome (for examples, see Murphy et al. 2018; Wang et al. 2020). In the present study, we specifically focused on the dissociation between the brain's language-specific system (with a particular focus on its reaction to semantic operation) and multiple-demand system through the lens of their positioning on the Margulies et al. principal gradient. Neuroimaging of healthy individuals and neuropsychological studies on aphasic patients have robustly demonstrated that a set of frontotemporal regions are selectively engaged during language processing (e.g., Fedorenko et al. 2011). These regions, collectively called the language network, are strongly left-lateralised and include the left inferior frontal gyrus (IFG) and a long stretch of the temporal cortex (encompassing its posterior, anterior, and ventral sections). They show selective response to language processing irrespective of the modality of language stimuli (text, speech, Braille) and the requirement of a task (provided the task is focused on linguistic aspects). Their reaction heightens when a task stipulates deeper language processing and declines when attention is steered towards non-linguistic stimuli, closely tracking the necessity to comprehend language (for review, see Fedorenko 2014). Language regions show reliable synchronised activity both in task-states and resting-state, and tend to be jointly susceptible to neurodegenerative diseases (e.g., primary progressive aphasia; Rogalski et al., 2011). Together, converging evidence has established this system's functionally specificity for language (particularly for comprehending linguistic meaning). The domain-specificity of the language system - i.e., its strong preferential reaction to language - is often contrasted with the domain-general nature of another set of brain regions. These regions, collectively called the multiple-demand network, are distributed in both hemispheres and include a broad swathe of frontoparietal regions (Duncan 2010; Duncan et al. 2020). They are broadly engaged by a diverse range of non-linguistic cognitive tasks (e.g., arithmetic, working memory, Stroop-type inhibition, mental rotation, etc.), in stark contrast with the language system that strongly favours language processing (Fedorenko et al. 2013). Intriguingly, while the role of the multiple-demand system is indispensable for performing a wide variety of cognitive tasks (particularly when tasks are cognitively taxing), it might have only an ancillary role in language processing. Its supplemental yet non-essential contribution to language tasks has been observed in contrastive patient research (Woolgar et al. 2018), as well as in its lack of ability to closely track language input (Blank and Fedorenko 2017). Together, multiple lines of evidence have suggested that these two neural networks are anatomically and functionally separable from each other.

In the present study, we used functional MRI to investigate task-evoked neural activity while orthogonally manipulating two factors: the type of cognitive operation one was required to perform (Semantic vs. Visuospatial) and the difficulty in attaining correct responses (Easy vs. Hard). With this 
2-by-2 factorial design, we identified two constellations of regions that differed drastically in their preferential tuning to tasks and in cerebral configuration. One was tuned to the domain-specific need of semantic operation, while the other was tuned to the domain-general need of more cognitive effort irrespective of stimuli/tasks. The two modules occupied largely separable cortical territories, with minimal anatomical overlaps. In addition, we identified cortical modules with specialised tuning to enhanced effort only in the semantic domain $v s$. those only in the visuospatial domain. We projected these modules onto the principal cortex gradient (Margulies et al., 2016) to explore whether they were couched in different tiers of the cortical hierarchy. To foreshadow our main findings, we found that the language-specific and multi-demand networks were situated at separate ranks of the gradient and exhibited qualitatively distinct trajectories in the manner they distributed along the gradient, suggesting their differential roles in the course of transitioning from perception to cognition. Importantly, when semantic processing became difficult, the brain reacted to this challenge by recruiting a specialised 'semantic-control' subsystem that was both functionally and anatomically distinct from the 'visuospatial-control' subsystem. The semantic-and visuospatial-control subsystems were specifically tuned to difficulty in their respective domain while insensitive to (even suppressed by) difficulty in the other domain. Moreover, the semantic-control subsystem's anatomical locus gave clues regarding how its unique functional profile arose - in line with recent findings by Wang et al. (2021), this subsystem occupied the intermediary cortical loci sandwiched between the multi-demand (executive-control) and default-mode (abstract cognition) networks. Its intermediary locus rendered the semantic-control system a hybrid middle-ground wherein the domain-specific need for resolving a semantic problem coalesced with the domain-general need for dedicating more cognitive resources, giving rise to its proclivity for semantic difficulty. This lends further support to previous findings for a specialised executive-control machinery for handling semantic information (e.g., Davey et al. 2016; Chiou et al. 2018; Jackson 2020). In the Discussion, we expound on how the principal gradient of cortical connectivity provides a framework that accommodates all these functionally distinct modules into a coherent, meaningful spectrum both at functional and anatomical levels.

\section{Methods}

\section{Participants.}

Twenty-five volunteers gave informed consent before the fMRI experiment. The sample consisted of 15 females and 10 males, with an average age $=28$ years-old and $\mathrm{SD}=6$. All volunteers speak English as their mother tongue and are right-handed. All of them completed a magnetic resonance imaging safety screening questionnaire before participating in the scanning session and reported not having any neurological or psychiatric condition. This study was reviewed and approved by the ethics board. 


\section{Design and stimuli.}

Participants completed eleven runs of echo-planar imaging in a single session. All of the functional MRI and behavioural results reported in the present study were acquired in the first run of scanning. The remaining ten runs of scanning, from Run 2 to Run 11, were designed for a separate project, and those data are not reported here. In the present study, we focused on the oddity detection experiment in which participants were required to identify either a semantic anomaly or a geometric anomaly from an array of four items. As illustrated in Figure 1A, in each trial, we presented a quadruplet of visual stimuli; one of the four was an 'oddball' that was either semantically or visuospatially inconsistent with the other three items. We used a 2-by-2 factorial design in which we orthogonally manipulated the types of stimuli (words $v s$. polyominoes) and the extent of difficulty (easy $v s$. hard), yielding four task-conditions. The overarching task requirement was identical in every condition participants were required to find out the oddity that differed from other items. However, words and polyominoes entailed different cognitive operations to achieve the same behavioural goal of identifying an oddball. Detecting a semantic oddity required comprehending the meaning of each constituent word and deriving an abstract conceptual relationship amongst them, whereas detecting a visuospatial oddity required analysing the visual features of each polyomino and mentally rotating them to derive their spatial relationship when necessary. A session began with the acquisition of each person's anatomical scan, followed by the oddity detection experiment (Run 1), and then a separate experiment (Run 2 - Run 11). To maintain continuous engagement in a cognitive state for a sufficient period, we used a block design. The experiment comprised 48 task-episodes (each of the four taskconditions contained 12 blocks; each block was 12-sec long, consisting of three trials), 47 inter-block intervals (each $1.5 \mathrm{sec}$ ), and a 1-sec blank at the final moment of the scan, yielding a total duration of $647.5 \mathrm{sec}$. Each trial began with a fixation cross $(0.1 \mathrm{sec})$, followed by a quadruplet of stimuli (3.9 $\mathrm{sec}$ ); the quadruplet consisted of either words or polyomino patterns, each bounded inside a square. Participants were instructed to respond as quickly as possible within the time-limit. Stimuli display and response collection were controlled using E-Prime (Psychology Software Tools). We fully counterbalanced the order in which the four task-conditions were presented so that each condition was equally likely to appear in every possible timeslot within the sequence (i.e., each condition was equally probable to precede or succeed any other condition), with stimuli randomly drawn from the designated stimuli-sets and shuffled across blocks. When performing the task, participants reacted by pressing one of the four designated buttons on an MR-compatible response-pad using their right hand. The oddball's location varied randomly trial-by-trial and was equally probable to appear in any of the four locations. All text stimuli were white colour, displayed on a black background; text stimuli were Arial typeface, 24-point in font size. Stimuli were displayed using an MRI-specialised LCD screen $(1,920 \times 1,080 ;$ NordicNeuroLab) and projected onto a set of coil-mounted device for viewing. 
For the semantic anomaly task, we constructed 72 'odd-one-out' quiz questions; 36 questions were designed for the Semantic-Easy condition, while 36 were designed for the Semantic-Hard condition. The complete list of the 72 questions is reported in Supplementary Materials. Each of the questions was presented only once to prevent dwindled neural reaction to repeated exposures. Each question contained a target (semantic oddball) and three foils. Questions of the two conditions differed on the degree of scrutiny required to differentiate semantic concepts. The Semantic-Easy questions were designed based on the following two rules: $(i)$ The semantic oddball belongs to a different basic-level linguistic category from the three foils. (ii) The three foils are semantically related to each other, while the oddball is unrelated to any of them. With these principles, we constructed a set of questions in which every semantic oddball easily stands out from the quadruplet as it has noticeably distinct features from any other words. For instance, in the quadruplet of 'Lizard, Piano, Flute, Drum', all of the three foils belong to the broad category of music instruments and are semantically unrelated to the reptile animals of lizard. The Semantic-Hard questions were designed with the following rules: (i) All of the four words in a quadruplet belong to the same basic-level category. (ii) The oddball is semantically related to the three foils and only differs from them on an idiosyncratic semantic attribute at the subordinate linguistic level. For example, in the quadruplet of 'Sprite, Gin, Vodka, Cider', all of the four word belongs to the broad category of beverages and are ingredients for making cocktail; however, Sprite is the only non-alcoholic drink. To further maximise the disparity between the Easy and Hard condition in the difficulty of semantic retrieval, we deliberately selected words with lower lexical frequency for the Hard condition than the Easy condition (frequncy per million words: Easy 5,499 $\pm 1,287$, Hard - 526 $\pm 134, p<0.001$; the corpus statistics based on Van Heuven et al. 2014).

For the visuospatial anomaly task, we created 72 polyomino patterns. These stimuli were used to construct 72 quadruplets (non-repeated); 36 were in the Visuospatial-Easy condition; the remaining were used in the Visuospatial-Hard condition. Example stimuli of the two conditions are illustrated in Figure 1A. Each polyomino pattern consists of a black background and 3-by-3 intersecting grids; within a pattern, five cells from the nine positions were tinted with different colours. As shown in Figure 1A, in the Visuospatial-Easy condition, the three foils have exactly identical configuration, orientation, and colours, while the oddball is saliently distinct from all other items (akin to the classic 'pop-out' phenomenon in the visual search literature; Treisman and Gelade 1980). By contrast, in the Visuospatial-Hard condition, the three foils have the same configuration and colours, but they are rotated to $0^{\circ}, 90^{\circ}, 270^{\circ}$ (essentially, they are the same stimulus shown in three different orientations). The oddball in the Visuospatial-Hard condition has a similar visual configuration to its accompanying foils and differs subtly on the spatial arrangement of one or two cells. 


\section{fMRI acquisition.}

MRI data was collected using a Siemens 3-Tesla PRISMA system. $\mathrm{T}_{1}$-weighted anatomical images were acquired using a 3D Magnetization Prepared RApid Gradient Echo (MPRAGE) sequence [repetition time $(\mathrm{TR})=2250 \mathrm{~ms}$; echo time $(\mathrm{TE})=3.02 \mathrm{~ms}$; inversion time $=900 \mathrm{~ms} ; 230 \mathrm{~Hz}$ per pixel; flip angle $=9^{\circ}$; field of view (FOV) $256 \times 256 \times 192 \mathrm{~mm}$; GRAPPA acceleration Factor 2]. Functional task-evoked data were obtained with a multi-echo multi-band (MEMB) blood oxygenation level dependent (BOLD)-weighted echo-planar imaging (EPI) pulse sequence. This MEMB sequence has the strengths that it acquired four functional volumes for each TR (multi-echo, enabling capturing signals that peaked at early and late echo-times that are often overlooked by conventional protocols) and simultaneously recorded two slices during the acquisition of each volume (multi-band, speeding up the acquisition rate). The parameters included: $\mathrm{TR}=1,792 \mathrm{~ms} ; \mathrm{TE}_{1}=13 \mathrm{~ms}, \mathrm{TE}_{2}=23.89 \mathrm{~ms}, \mathrm{TE}_{3}$ $=34.78 \mathrm{~ms}, \mathrm{TE}_{4}=45.67 \mathrm{~ms}$; flip angle $=75^{\circ} ; \mathrm{FOV}=192 \mathrm{~mm} \times 192 \mathrm{~mm}$, MB-Factor $=2$, in-plane acceleration $=3$. Each EPI volume consisted of 46 axial slices in descending order $(3 \times 3 \mathrm{~mm}$; starting concomitantly from the top and middle slice) covering the whole brain (FOV $=240 \times 240 \times 138 \mathrm{~mm})$. For the present study, a series of 362 functional volumes were acquired for the oddity detection task.

\section{Pre-processing.}

All raw DICOM data were converted to NifTi format using dcm2niix. The $T_{1}$ structural images were processed using the standard processing pipeline of the FSL package's 'fsl_anat' function (Ver5.0.11; https://fsl.fmrib.ox.ac.uk/). This pipeline involves these sequential six steps: (i) Reorient images to standard MNI space ('fslreorient2std'), (ii) automatically crop image to remove the neck ('robustfov'), (iii) bias-field correction to fix field inhomogeneity ('fast'), (iv) registration into the MNI space ('flirt' and 'fnirt'), ( $v$ ) brain extraction (using 'fnirt' nonlinear method) and (vi) tissue-type segmentation to separate white-/grey-matter and other structures ('fast'). Each $\mathrm{T}_{1}$-image was individually inspected for accuracy after being normalised into the MNI space. The functional EPI data were pre-processed using a combination of tools in FSL, AFNI (Ver18.3.03; https://afni.nimh.nih.gov/), and a specialised Python package to perform TE-dependent analysis (Kundu et al. 2012; Kundu et al. 2013; Kundu et al. 2017). Despiked ('3dDespike'), slice-time corrected ('3dTshift', matched to the middle slice), and realigned ('3dvolreg') images were submitted to the "tedana" toolbox, which took the time-series data from all of the four TEs and decomposed the resulting data into BOLD-signals and noises (nonBOLD components). Specifically, decomposition was based on whether a signal series depended on the four echo-times - with the strength of multiple echo-times, the algorithm was able to tell apart noises that fluctuated randomly or independently of the timings of four TEs (e.g., scanner's drift, cardiac/respiratory noises, head motion) from signals that systematically varied with the timings of TEs (e.g., the functional data of BOLD). Data of the four echo-times were then optimally integrated, 
weighted based on the intensity of $\mathrm{T}_{2} *$ signal in each voxel and separated from the TE-independent/ non-BLOD noises (Kundu et al. 2017). Finally, the optimally-combined images were co-registered into each individual's $\mathrm{T}_{1}$ structural scan (using FSL's 'flirt'), normalised to the standard MNI space (using FSL's 'fnirt' warps and 'flirt' transform), and smoothed with a 6 mm FHWM Gaussian kernel.

\section{General linear model and psychophysiological-interaction connectivity.}

The SPM12 package (https://www.fil.ion.ucl.ac.uk/spm/software/spm12/) was used to construct a general linear model (GLM) for subsequent analyses. For each participant, the onset times and durations of every task-episode were used to create an experimental-design matrix. Each individual's design matrix was convolved with a canonical haemodynamic response function. We included each participant's reaction times (RTs) as a parametric modulator that were attached to each task regressor, allowing us to take into account neural activities driven by task difficulty or cognitive effort when assessing the effects of experimental manipulation. Contrast images from the fixed-effect model at individual-level ( $1^{\text {st }}-$ level$)$ were submitted to the random-effect model at the group-level ( $2^{\text {nd }}-$ level). We statistically thresholded the whole-brain interrogation GLM results at $p<0.05$ (FWE-corrected for multiple comparisons) for clusters and $p<0.001$ for voxel intensity.

To investigate how context-dependent connectivity to the inferior frontal gyrus (IFG) altered between linguistic and non-linguistic situations, we used SPM12 to perform a psychophysiological-interaction (PPI) analysis. The IFG, also known as the Broca's area, has been implicated in a broad range of language-related processes (for review, Fedorenko and Blank 2020), particularly when participants allocated greater amount of cognitive resources to solve a semantic problem (e.g., Chiou et al. 2018). We used the left IFG as a region of interest (ROI) and individually defined its locus for each person, guided using the group-level peak activation coordinate $(x=-44, y=24, z=-2)$ from the contrast of 'Semantic-Hard > Semantic-Easy' (which identified brain regions associated with higher difficulty of semantic processing). For each individual, we pinpointed the (Semantic Hard > Easy) local maxima of IFG activation nearest to the group-level peak coordinate (searched within the scope of $8 \mathrm{~mm}$ radius) and set it as the 'seed' of PPI connectivity. At each individual's IFG peak, we created a spherical ROI (radius $=6 \mathrm{~mm}$ ) and extracted the first eigenvariate in the sphere using SPM12's built-in algorithm. The eigenvariate was a proxy of the seed's underlying neural/physiological activities. It was then convolved with the psychological factor (the contrast of cognitive states: Semantic vs. Visuospatial). This generated the interaction term - the psychophysiological/PPI factor that denoted changes in connectivity with the IFG seed as a function of switching between task-conditions. These three factors - the psychological, physiological, and PPI - were used to construct a GLM for whole-brain search. We focused on the PPI regressor to identify brain regions whose neural connectivity with the left IFG 
was modulated as a function of Semantic vs. Visuospatial context. Statistical thresholds were the same as those for the GLM analysis $-p<0.001$ for voxel's activation strength (for cluster formation) and $p<0.05$ (FWE-corrected for cluster-level multiple comparisons).

\section{Cortical gradient analysis.}

To understand how the neural activity triggered by different task-conditions were couched within the macroscale architecture of whole-brain connectivity, we projected various fMRI results onto the principal (first) gradient of Margulies et al.'s (2016) hierarchical framework of brain organisation. The methodological details of deriving the cortical gradients were reported in the original study. Here we summarised their main analytical steps: Using the resting-state fMRI data of 820 participants from the Human Connectome Project combined with different nonlinear dimension-reduction methods (e.g., Laplacian eigenmaps and diffusion map embedding), Margulies et al. analytically reduced the complexity of connectivity into two dimensions that succinctly delineated the majority of variance regarding how regions are functionally linked together. The principal gradient, which explained the most extent of variance, was anchored, on one end of the spectrum, at the primary sensory-motoric regions that directly receive input from the external world and generate a response to interact with the environment; on the other end of the spectrum, the gradient was anchored at the default-mode regions that are involved in abstract cognition. The original gradient structure assigned each voxel of the brain a value between 0 and 100, relating to where it fell on the gradient $(0=$ sensory-motor end; $100=$ default-mode end). For our analysis, this gradient was divided into 20 bins, with voxels within each five-percentile bin lumped together (for example, all voxels with a value between 0 and 5 were grouped in Bin-1, and all voxels ranged 6-10 were grouped within Bin-2, etc.; each bin contained nearly identical number of voxels - range: 6,431 -6,441; mean \pm SD: 6,436 \pm 2 ) (for precedents of this approach, see Murphy et al. 2018; Murphy et al. 2019; Lanzoni et al. 2020; Wang et al. 2020). Next, we used each of the 20 five-percentile bins as a region of interest (ROI) and extracted activation amplitudes (from GLM) and connectivity strengths (from PPI), allowing us to investigate how the neural correlates were distributed across the gradient. The original gradient data of Margulies et al. (2016) are publicly available online (https://www.neuroconnlab.org/data/index.html).

\section{Results.}

\section{Behavioural performance.}

The overarching behavioural goal was identical in every task-condition (identifying an oddball from a quadruplet) but the stimuli and required operations differed between tasks. Figure 1C illustrates the group-level averages of accuracy rate and reaction time. To ascertain whether our manipulation effectively modulated the difficulty of semantic and spatial processing, we performed $a$ priori tests 
to compare performance under the Easy and Hard conditions for the Semantic and Visuospatial tasks. The effects were reliably detected in both tasks - in the Semantic task, identifying a semantic oddball was less accurate $\left(t_{(24)}=13.91, p<0.001\right)$ and slower $\left(t_{(24)}=-13.70, p<0.001\right)$ in the Hard condition (greater difficulty in accessing and differentiating semantics due to lower lexical frequency and more overlaps in semantic attributes) than the Easy condition (higher frequency, less overlap in attributes); in the Visuospatial task, identifying a visual oddball was also less accurate $\left(t_{(24)}=14.08, p<0.001\right)$ and slower $\left(t_{(24)}=-21.34, p<0.001\right)$ in the Hard condition (wherein the oddball could only be found through careful inspection) than the Easy condition (wherein the oddballs 'popped out' due to their clearly distinctive features). These results confirmed the efficacy of our difficulty manipulation for each task. Next, we performed a 2-by-2 repeated-measure ANOVA, with Task and Difficulty being within-participant variables. In the results of reaction time, there was significant interaction between Task and Difficulty $\left(F_{(1,24)}=217.38, p<0.001, \eta^{2} \mathrm{p}=0.90\right)$. Post-hoc tests were performed to dissect the source of this interaction - while the Semantic-Hard and the Visuospatial-Hard conditions yielded comparable reaction time (Semantic-Hard $v s$. Visuospatial-Hard: $p=0.28, n . s$. ), reaction time was faster in the Visuospatial-Easy than in the Semantic-Easy condition $(p<0.001)$. The same $2 \times 2$ interaction was not significant in accuracy rate $(F<1)$. Taken together, behavioural results indicated that our experimental design was effective in manipulating difficulty for both semantic and visuospatial domains, and which warranted further investigation into the neural substrates that underlay domain-general, semantic-specific, and visuospatial-specific difficulties.

\section{fMRI analysis on task-evoked activation, functional connectivity, and parametric modulation.}

Multiple-demand system. It has been reliably demonstrated that the brain recruits a generic machinery, a constellation of frontoparietal areas known as the 'multiple-demand' system, when confronted with a diverse range of cognitively challenging situations (for review, Duncan et al. 2020). We began with a targeted examination on whether difficulty manipulations for the Semantic and Visuospatial tasks were both effective in raising the response amplitude of this system. Using 10 cortical parcels in the frontal and parietal cortex that have been formerly used as ROIs of the multipledemand system (e.g., Blank and Fedorenko 2017; Shashidhara et al. 2019; Shashidhara et al. 2020), we separately inspected the contrasts of 'Hard > Easy' for the Semantic and Visuospatial task in these regions. Results showed that greater difficulty of these two tasks was both effective in augmenting bilateral activation of nearly every multiple-demand ROI (see Supplemental Analysis 1 in Supplemental Materials for statistics). Within the system, the effect of semantic difficulty showed a trend of left-lateralisation, whereas the effect of spatial difficulty was equally strong in both hemispheres. Closer inspection revealed that semantic difficulty selectively enhanced the prefrontal and cingulo-opercular subregions of the system while minimally engaging the subregions related to 
saccadic movement and spatial attention. Moreover, while both types of difficulty reliably amplified the reaction of multi-demand network, visuospatial difficulty triggered generally grater activation than semantic difficulty. These results support some extent of generality of the system across semantic and spatial domains while highlighting the nuances that cognitive effort devoted to different domains could entail domain-specific machineries (outside the multiple-demand network) or preferentially engage some subregions within the network while sparing others. In the present section, we focus on domain-generality using a whole-brain GLM search to identify regions with generic sensitivity to heightened cognitive effort (regardless of tasks). In the later section of 'semantic-control', we will elaborate on the domain-specific machineries.

As illustrated in Figure 2(A), we interrogated the entire brain, comparing Semantic-Hard and Visuospatial-Hard against the two Easy conditions. This contrast revealed a characteristic pattern of the multiple-demand system. In response to increased difficulty, activation intensified in the bilateral frontoparietal regions well-documented in the executive-control literature (e.g., Cole et al. 2013; Duncan et al. 2020). The reverse contrast - regions more active for Easy conditions than Hard ones - revealed heightened activation of the posterior cingulate cortex and ventromedial prefrontal cortex that are known as 'midline cores' of the default-mode network (Andrews-Hanna et al. 2010). Next, we investigated how the multiple-demand system was couched along the 20 tiers of a 'sensorimotor to cognition' spectrum by projecting the unthresholded whole-brain map of 'Hard > Easy' responses onto the principal cortical gradient of Margulies et al. (2016). Under this framework, the system was characterised by a 'twin-peak' configuration along the spectrum. Statistics confirmed that the generic difficulty effect indeed varied along the gradient $\left(F_{(19,456)}=30.09, p<0.001\right)$. Shown in Figure 2(B), polynomial regression was used to characterise the trajectory with which the multiple-demand system was distributed across the spectrum. It is evident that regions situated on the polar extremes of the spectrum (the primary somatosensory-motoric and default-mode regions) did not contribute to the neural processing for difficulty-triggered operations while regions situated in the intermediate tiers were heavily involved. The most responsive tiers (Tier $4-6$ ) to the Hard conditions comprised regions known to underpin oculomotor control and visual attention, such as the frontal eye fields and posterior parietal lobules. Following the primary peak, the next peak consisted of Tier $12-14$, which contained a large proportion of the dorsolateral prefrontal cortex known to support executive function. Together, situating the multiple-demand system along the principal gradient provides the insight that executive function relies on intermediary regions between extreme positions of the cortical hierarchy, higher than the sensorimotor system yet lower than the default-mode system. Such a juxtaposition is consistent with its postulated role as a mediator that facilitates the real-time update and the crosstalk between lower- and higher-order systems (Duncan 2010; Duncan et al. 2020). 
Language-specific system. Next, we focused on brain regions that are sensitive to the GLM contrast between Semantic and Visuospatial tasks, regardless of difficulty level. Thus, we focused on the main effect of Task, comparing Semantic-Easy and Semantic-Hard against the two Visuospatial conditions. As illustrated in Figure 3(A), this contrast revealed a set of strongly left-lateralised regions typical of the configuration of language-specific system (Fedorenko et al. 2011). Relative to the requirement of processing meaningless polyominoes, the Semantic task elicited greater activation of the left IFG, encompassing its anterior and posterior subparts and extending to the dorsolateral prefrontal cortex. The left IFG, also known as 'Broca's area', is known for its contribution to various aspects of language-related functions (semantic, phonological, syntactic; also see Fedorenko and Blank 2020 for functional differentiation between the left IFG's subregions). The Semantic task also activated the superior temporal gyrus (STG) known for its role in spoken and written language (Wilson et al. 2018); the STG cluster included anterior and posterior subparts, and extended ventrally to the entorhinal cortex/hippocampus, which is implicated in representing semantic meaning (Clarke and Tyler 2015). To more specifically examine whether the language-specific and multiple-demand systems reacted differently to the Semantic-oddity task, we confined the scope of inspection to the functional ROIs of the language-specific network (Fedorenko et al. 2011) and the multiple-demand network (Fedorenko et al. 2013) defined in prior research. Statistics are reported in Supplemental Analysis 2 - we found that, the language network showed significantly greater activation for the Semantic task than Visuospatial task, whereas the multiple-demand network exhibited the reverse pattern. However, it is noteworthy that the language network's preference for the Semantic over Visuospatial task was approximately 2-times bigger than the multi-demand network's preference for the Visuospatial over Semantic task. Such discrepancies have two implications: (i) consistent with previous findings (e.g., Blank and Fedorenko 2017), the language network has specific 'fondness' for linguistic stimuli over other categories; (ii) compared with the high specificity of language system, the multi-demand system has heterogeneous tastes; although the multi-demand network preferred visuospatial over semantic processes, this system was still moderately responsive to semantic difficulty (note that the reaction of multi-demand system to semantic difficulty might reflect intensified executive-control for solving semantic problems, rather than semantics per se; see Wehbe et al. 2021 for discussion). Figure 3(B) shows where the contrast of 'Semantic vs. Visuospatial' was loaded onto the cortical gradient. Semantic and visuospatial processes recruited different cortical tiers $\left(F_{(19,456)}=60.12, p<0.001\right)$ : the Semantic task relied heavily on higher-order tiers on the abstract-cognitive end, whereas the Visuospatial task relied heavily on lower-order tiers on the sensory-motoric end (particularly Tier 3 -6 that contain regions related to saccade and visual attention). 
Semantic-control system. We directly compared whether the brain employed separate mechanisms to tackle cognitive burden specifically in the semantic or visuospatial domain. As shown in Figure 4(A), activities driven by semantic difficulty (Semantic-Hard > Semantic-Easy) are colour-coded in yellow, whereas those driven by perceptual difficulty (Visuospatial-Hard > Visuospatial-Easy) are in magenta. These two types of difficulty-triggered activities had both conjunctions and dissociations. Frontoparietal regions known to be heavily implicated in spatial attention and oculomotor control, including the frontal eye fields, posterior parietal lobules, and large swaths of the visual cortex, were exclusively engaged by spatial difficulty. These 'exclusively spatial' regions largely fell within the realm of the multiple-demand system (Fedorenko et al. 2013) and overlapped extensively with the 'dorsal-attention' system of the resting-state literature (Yeo et al. 2011). By contrast, the activities exclusively driven by semantic difficulty formed a hybrid configuration comprising elements of the default-mode, language-specific, and multiple-demand systems. Specifically, it contained the 'core' sections of the default-mode system (the ventro/dorsomedial prefrontal cortex, retrosplenial cortex, and left angular gyrus known to be implicated in introspective/mnemonic processes; Andrews-Hanna et al. 2010). It also contained sections of the dorsolateral prefrontal cortex, which closely abutted the multi-demand system and are known to contribute to verbal working memory (Barbey et al. 2013). Importantly, it contained regions known for their contribution to semantically challenging situations - the left IFG and the left posterior middle temporal gyrus (collectively, these left-lateralised regions have been called the 'semantic-control' system; see Noonan et al. 2013; Jackson 2020). These 'semantic-control' regions overlapped minimally with the multiple-demand network (Fedorenko et al. 2013) and fell largely within the territory of the language-specific network (Fedorenko et al. 2011). However, rather than underpinning the contents of semantics per se, these 'semantic-control' regions have been repeatedly shown to underpin the goal-directed retrieval and manipulation of semantics specifically (for comprehensive review, see Lambon Ralph et al. 2017). While semantic and spatial difficulties dissociated on their respective domain-specific regions as discussed above, they also recruited overlapped cortical sections in various parts of the multi-demand network (orange clusters in 4A: the cingulo-opercular subnetwork, bilateral middle frontal gyri, and a small section of the left intraparietal sulcus), corroborating the ROI analysis within the multi-demand system reported earlier.

The hybrid configuration of the 'semantic difficulty' activation (i.e., partially default-mode, partially multiple-demand, largely within the broad language-specific network) suggests a possibility that this machinery might be juxtaposed between the default-mode and multiple-demand systems ( $c f$. Davey et al. 2016; Wang et al. 2020). As a result of this juxtaposition, the 'semantic-control' mechanism might, functionally speaking, intervene in the processes of directing executive-control resources to 
abstract cognition. To investigate this, we compared semantic difficulty with 'perceptual easiness' (Visuospatial-Easy > Visuospatial-Hard - reflecting less executive-control and more automaticity). Behavioural data revealed that the Visuospatial-Easy condition was the least demanding one among all conditions. Given the well-established observations that default-mode activity tends to decline in cognitively difficult contexts and to elevate in easy ones (e.g., Raichle et al. 2001), we anticipated that contrasting the Visuospatial-Easy against Hard would reveal the default network. Consistent with this speculation, default-mode activation intensified during the Visuospatial-Easy condition: the most rostral sector of the medial prefrontal cortex, the retrosplenial and posterior cingulate cortices, and the bilateral angular gyri (cyan in 4B). Crucially, topographical arrangement of the default-mode, semantic-control, and multiple-demand systems manifested as an orderly transition, with the semantic-control regions juxtaposed in the middle zones. Inspection on Figure 4(A) and (B) showed that this juxtaposition was dispersed in various parts of the brain: In the medial surface, there was a posterior-to-anterior transition, from multiple-demand being caudal (magenta: midcingulate cortex), through semantic-control in the middle (yellow: dorsomedial prefrontal cortex), to default-mode being rostral (cyan: rostromeidal prefrontal cortex). This graded layout was also seen in the prefrontal/parietal lobes, unfolding from dorsal (multiple-demand: frontal eye field/superior parietal lobe) to ventral regions (default-mode: frontal pole/angular gyrus), with semantic-control occupying intermediary zones. In all of these transitions, the semantic-control clusters were situated in the middle-ground between the multiple-demand and default-mode systems.

The intermediary position of semantic-control system became even more obvious when we projected the whole-brain responses to semantic difficulty, perceptual difficulty, and perceptual easiness onto the cortical gradient. As shown in Figure 5, the low- to middle-tiers (the range shaded with blue, including many subregions of the multiple-demand system) were most active for perceptual difficulty, followed by semantic-control, and least for perceptual easiness. By contrast, the three topmost tiers (the range shaded with green, primarily containing subregions of the default-mode system) were most responsive to perceptual easiness, followed by semantic-control, and least to perceptual difficulty. Intriguingly, on Tier 15 and 16 (shaded with orange; these are the tiers wherein most regions of the language system were situated), the 'semantic difficulty' contrast elicited reliably greater activation than the other two contrasts. Statistics confirmed that different ranges of the cortical tiers indeed favoured different types of difficulty $\left(F_{(19,456)}=48.43, p<0.001\right)$. Comparing between the 20 tiers revealed that the ranking of tiers most sensitive to semantic-control is also in the intermediary levels on the cortical hierarchy - higher than sensory-motoric and multiple-demand regions but lower than default-mode regions. This potentially explains why the neural substrates of semantic-control occupied the cortical zones sandwiched between the multiple-demand and default-mode networks. 
bioRxiv preprint doi: https://doi.org/10.1101/2021.11.26.470178; this version posted November 26, 2021. The copyright holder for this preprint (which was not certified by peer review) is the author/funder, who has granted bioRxiv a license to display the preprint in perpetuity. It is made available under aCC-BY-NC-ND 4.0 International license.

Solving a difficult semantic problem required both more attentional resources to visual stimuli (text) and deeper semantic processing of abstract meaning, potentially explaining how its neural substrates were configured in intermediary positions on the cortical spectrum.

\section{PPI analysis on context-reliant connectivity to the IFG and its distribution along the gradient.}

The left IFG, a region known for its contribution to semantic operation, has an intriguing profile in its functional reaction to different task-situations and affiliation with different networks. The left IFG was significantly more active for Semantic-Hard than Semantic-Easy while also significantly more active for Visuospatial-Easy than Visuospatial-Hard (Figure 4B). This mixed picture indicated that, unlike regions of the multiple-demand system that were purely driven by difficulty level, the reaction of left IFG was specific to greater difficulty only in the semantic domain. While it was active both for semantic difficulty and perceptual easiness ( $p s<0.001)$, both its anterior and posterior sections (pars orbitalis and triangularis) were more active for semantic difficulty than perceptual easiness (both $p$ s $<0.05$; also see Supplemental Analysis 3 that displayed the whole-brain interaction pattern of 'semantic difficulty-by-perceptual easiness'). This is consistent with prior findings that this area was more active during relaxing rest-intervals than difficult perceptual tasks (Chiou et al. 2020) while also more active for difficult semantic decisions than easy ones (Chiou et al. 2018). Connectivitywise, the left IFG was categorised as a component of the default-mode network (Yeo et al. 2011); however, it was also found to be reliably connected with various semantics-specific regions of the language network (Jackson et al. 2015) and some sections of the multiple-demand system (Humphreys and Lambon Ralph 2017). These results together suggest that the left IFG might serve as a 'switchboard operator' mediating the conversation between different specialised systems, which gave rise to its mixed functional profile. To further investigate this, we performed a PPI analysis to examine how neural connectivity with the left IFG altered as a result of our experimental contexts.

As illustrated in Figure 6(A), we placed the seed of connectivity at each individual's IFG peak activity for the 'semantic difficulty' contrast, and scrutinised across the whole-brain to identify voxels whose connectivity with the seed varied between the Semantic and Visuospatial contexts. This analysis uncovered a landscape of contextually-dependent connectivity that constituted an inverse pattern from the original GLM-contrast of Semantic vs. Visuospatial. Specifically, we found that during the Semantic condition, the left IFG was more tightly coupled with the bilateral multiple-demand regions and visual cortex; by contrast, during the Visuospatial condition, it was more connected with the default-mode system. This pattern is the exact antithesis of what the GLM results showed - in a direct comparison, the multiple-demand system and visual cortex were more active for the Visuospatial task, whereas the default-mode system (as well as the language-specific system) were more active for the 
Semantic task. These results suggest that when the brain is confronted with a challenge that interfaced between perception (i.e., reading visual text that conveyed meaning) and semantics (i.e., scrutinising their conceptual relationship to discover a semantic oddity), the left IFG played an intermediary role that facilitated the communication between multiple-demand and language-specific/default-mode systems ( $c f$. Davey et al. 2016). However, when the cross-talk between systems was not necessary (such as resting-state or the Visuospatial task wherein the stimuli conveyed no semantic meaning), the left IFG was normally aligned with zones preferentially tuned to mnemonic/linguistic processes (i.e., default-mode and language-related areas), consistent with prior literature (e.g., Yeo et al. 2011).

Projecting the whole-brain PPI parameters onto the brain's principal organisational gradient offered further evidence for the left IFG's liaison. As Figure 6(B) shows, areas on lower end of the continuum (specifically, Tier 4-6, which included the 'dorsal-attention' subregions of multiple-demand system) were more connected with the IFG-seed during the Semantic task whereas areas on higher tiers (particularly the three topmost tiers that contained primarily default-mode regions) were more connected with the seed during the Visuospatial task. Statistics confirmed that the contextual change of Semantic $v s$. Visuospatial significantly drove the left IFG to connect with different cortical tiers $\left(F_{(19,456)}=15.84, p<0.001\right)$. This pattern is consistent with the interpretations that $(i)$ there was an overall intensified dialogue between the left IFG and multiple-demand system when the context entailed deriving semantic meaning from perceptual input; (ii) while the multiple-demand system served as the brain's generic instrument to tackle cognitively laborious tasks, the semantic-control system was additionally recruited to interface executive-control with semantic contents.

\section{Parametric modulation and replication using the data of Humphreys \& Lambon Ralph (2017).}

In addition to the four task-conditions as regressors in the GLM (which allowed us to examine the brain through categorical/discrete contrasts between conditions), reaction times (RTs) were also included as parametric modulators to estimate the impact of RT fluctuation on neural activity (which allowed us to identify brain regions that tracked behaviour in a continuous manner). Note that, in this parametric analysis, we specifically assessed the net effect of RTs while the impact of task-conditions was statistically partial-out (i.e., after excluding the influence of conditions, we scrutinised whether residual variance could be explained by RTs and where in the brain this would manifest). As shown in Figure 7(A), this analysis revealed four different aspects of the brain's control machinery: $(\boldsymbol{i})$ Domain-general intensification: the activation of multiple-demand network intensified with longer RTs irrespective of task-conditions; such domain-general modulatory effect was observed in the middle frontal gyrus, insular cortex, frontal eye field, bilateral posterior/superior parietal lobules, and the visual cortex. (ii) Domain-general suppression: two midline areas of the default-mode network - 
the medial prefrontal and posterior cingulate cortices - became more active when RTs were shorter irrespective of tasks, suggesting that these midline default-mode 'cores' generally prefer automaticity to cognitive effort. (iii) Specificity to semantic control: the left IFG and its neighbouring left middle frontal gyrus were significantly more positively modulated by semantic RTs than visuospatial RTs, suggesting these regions' preference for devoting more cognitive resources to semantic processing than visuospatial processing. (iv) Specificity to visuospatial control: the dorsal-attention system (the bilateral superior/posterior parietal areas) and visual cortex were significantly more modulated by visuospatial RTs than semantic RTs, suggesting these regions' sensitivity to heightened demands in the visuospatial domain, relative to the semantic domain. The parametric analysis complements the findings of direct contrasts between task-conditions, and highlights both domain-generality and domain-specificity of the brain's control mechanisms.

We revisited the dataset of Humphreys and Lambon Ralph (2017) and found a strikingly consistent pattern of domain-generality and domain-specificity in this previous dataset. In this previous study, participants were required to either judge semantic relatedness or compare visual shapes, and difficulty level (easy $v s$. hard) was manipulated for each task, yielding a factorial design akin to the present study. As shown in Figure 7(B), in the domain-general contrasts of easy vs. hard, areas of the multi-demand network were more active for the hard conditions, whereas the default-mode network was more active for the easy conditions ${ }^{1}$. In the domain-specific comparisons, the left IFG was comparatively more active for semantic demands, whereas the dorsal-attention system was more active for visuospatial demands. Replicated across multiple analyses and two independent datasets, we found a highly consistent pattern - the domain-general machinery (invariant to stimuli/tasks) was supplemented by additional, context-dependent domain-specific machineries to tackle a challenge.

\section{Discussion}

Decades of research on human brain mapping have reliably demonstrated that the cerebral cortex comprises a set of functionally specialised networks that tile across the cortical sheet, forming a tapestry-like overall configuration. Some of the networks are organised in well-defined and circumscribed locality (e.g., the somatosensory system, visual system, and auditory system; all of these systems occupy a single contiguous expanse of cortex that can be demarcated with boundaries),

\footnotetext{
${ }^{1}$ Although the default-mode network generally tends to be more active for cognitively less effortful contexts, it is an oversimplification to define this network as a 'task-negative' system. While in the present study we found that the default network became less active when confronted with semantic difficulty and visuospatial difficulty, it has been shown that this network showed enhanced activation for behaviourally more difficult processes in the domain of episodic memory (Murphy et al. 2018; Murphy et al. 2019). It has been suggested that the functional goal of default network is to sustain introspective processes, inducing episodic memory and conscious thoughts (Smallwood et al. 2021).
} 
whereas other networks are organised in a widely distributed manner with long-range connections to maintain the communication between componential regions that are situated in different lobes (e.g., the language-specific system, multiple-demand system, and default-mode system; all of these systems comprise distant regions widely dispersed in different lobes). In a recent study by Margulies $e t$ al. (2016), the authors studied the factors that drove the spatial layout of brain systems and found a macroscale architecture that explained the topographical placement. A gradient-like transition, which represented the workflow of information processing from sensory-motoric to abstract-cognitive, accounted for the largest share of the brain's connectivity. An orderly arrangement of topographical transition, from lower- to higher-order systems, could be seen in multiple places of the brain, suggesting an overarching organisational principle that governs how different functional systems appear in their canonical positions. In the present study, we used fMRI to investigate how the multiple-demand and language-specific systems could be accommodated in the macroscale structure of a 'sensorimotor-to-cognition' spectrum. We found that the multiple-demand network was situated on low to middle tiers of the gradient and exhibited a 'twin-peak' configuration (with regions of the 'dorsal-attention' subnetwork constituting the peak at lower tiers and regions of the prefrontal and cingulo-opercular cortices constituting the peak at mid-range tiers; these subnetworks jointly formed the multiple-demand system). By contrast, the language system was situated on the topmost few tiers and often juxtaposed with regions of the default-mode system. Critically, when semantic processing became difficult, the brain reacted by additionally recruiting a specialised 'semantic-control' system that was both functionally and anatomically separable from the multiple-demand system. Unlike the multiple-demand system that was tuned to miscellaneous domains, the semantic-control system was specifically tuned to language-related difficulty while insensitive to difficulties in other domains. The gradient analysis revealed how the anatomical locus of the semantic-control system is suited to its specialised tuning to semantic difficulty - this system occupied the intermediary loci between multiple-demand and default-mode networks, consistent with previous conclusions on this system based on different analyses (Davey et al. 2016; Wang et al. 2021). Such intermediate locations rendered the semantic-control system a hybrid middle-ground wherein the domain-specific need for resolving a semantic problem coalesced with the domain-general need for enhanced cognitive effort, giving rise to its domain-specific tuning to semantic difficulty. Furthermore, the PPI connectivity evidence showed that the left IFG is a key site of the semantic-control system that mediated the crosstalk between regions on the low/middle tiers (multiple-demand system and visual cortex) and regions on the high tiers (language-related and default-mode regions). Importantly, converging results were ascertained using various analytical approaches - direct GLM contrasts of task-conditions, PPI connectivity, parametric modulation of RTs, and replicated in an independent dataset of Humphreys and Lambon Ralph (2017). In the discussion below, we focused on two crucial issues that are relevant 
to our findings $-(i)$ the functional fractionation within the broad language system (ii) the unique insight about the brain's functional architecture that the gradient approach provides.

\section{The functional fractionation within the broad language system.}

Built on a wealth of converging evidence with different methodological approaches accumulated over the past two decades, we recently proposed a framework termed controlled semantic cognition (CSC) to account for how the brain underpins the ability to flexibly use semantic knowledge (Lambon Ralph et al. 2017). The CSC framework was distilled via decades of neuroimaging data of neurologically healthy individuals and neuropsychology data of two subtypes of language-related disorders (i.e., semantic dementia vs. semantic aphasia) and validated with computational modelling and neurostimulation to ascertain the causality link from brain areas to functions (some recent findings: Chiou and Lambon Ralph 2016; Jung and Lambon Ralph 2016; Rice et al. 2018; Jackson et al. 2021; for reviews of our data over the past decades, see Lambon Ralph et al., 2017; Jefferies et al. 2020). Under this framework, the ability of adaptively using semantic knowledge according to different contextual demands relies on two separable apparatuses: $(i)$ the brain system that represents the contents of semantic knowledge per se, (ii) the brain system that controls the context-dependent flexible retrieval and recombination of semantic meaning. The system specialised for representing semantics itself relies on a 'hub-and-spoke' configuration of ensemble neural activation, with the anterior temporal lobe (ATL) being the 'hub' and widely distributed cortical zones in different modality-specific cortices being the spokes (e.g., see Chiou and Lambon Ralph 2019 for example evidence about the neural substrates of action/place knowledge). By contrast, the system for controlling the flexible manipulation of semantics relies on a specialised 'semantic-control' system, with the left IFG and posterior middle temporal gyrus (pMTG) being its key underpinning (see Jackson 2020 for review about the neural processing for semantic-control; also see the present results of 'Semantic-Hard > Semantic-Easy' in Figure 4 that highlights left IFG and pMTG activation). Evidence accumulated over the past decades suggests that these two sub-systems are sensitive to different types of task manipulations and associated with different profiles of behavioural deficits following focal damage to respective brain areas.

Interestingly, the broad semantic system (combining its representation and control sub-systems) that we have been focused on has substantive overlap with the language-specific system that Fedorenko and colleagues have identified (particularly notable is the overlap in the left IFG and pMTG). However, we also noticed discrepancies - whereas we have repeatedly observed ventral ATL activation for semantic tasks, it was not reliably found in the works by Fedorenko and colleagues (see Fedorenko et al. 2020 for discussion about the absence of ATL activity). Two methodological issues 
are related to the commonalities and differences: First, both groups have been able to detect robust activation of the 'semantic-control' regions (i.e., the left IFG plus pMTG) when contrasting meaningful stimuli (words or speech) with meaningless stimuli (non-words or noise) despite different tasks and stimuli. This highlights the tuning of these areas to language/semantic-related processing, invariant to input modality. However, our results further emphasise these areas' selective preference for difficult semantic operations - whereas the difficulty of non-linguistic processing did not activate (even suppressed) these regions, the difficulty of linguistic processing further augmented them (on top of their preference for semantic over visuospatial processing). Second, the choice MRI acquisition protocols and contrasting baseline make a huge difference regarding whether ventral ATL activation is detectable. As has been discussed in a meta-analysis (Visser et al. 2010), many regions involved in semantic processing show a propensity to remain highly active during resting interval (which is consistent with people's subjective experience that the mind is still engaged by meaningful thoughts even when they passively fixate at a blank screen), which renders passive rest an inadequate baseline for contrasting with language-related conditions. In addition, due to its proximity to air-filled sinuses the ATL tends to suffer from severe signal dropout. To combat the signal-dropout and baseline issues, we have been employing multiple-echo protocols (which has been demonstrated to improve the signal-to-noise ratio around the ventral ATL, see Halai et al. 2014) and using meaningless stimuli and non-semantic tasks (rather than passive rest) as contrastive baselines. Interestingly, while the ATL strongly favours semantic stimuli over meaningless ones, it is generally insensitive to different difficulty levels of semantic operation (or, put differently, not as sensitive to difficulty levels as those areas specifically tuned to semantic-control; this pattern was found not only in the present study but also in Hoffman et al. 2015). Taken together, while the language system shows a specialised tuning to linguistic stimuli, further investigation into the functional fractionation within this system is a fruitful direction for future research.

\section{Situating functional networks along the gradient of macroscale structure.}

The principal organisational gradient of the human brain provides a comprehensive framework to explain a broad range of psychophysical and neuroimaging data. For example, it has been shown that task-contexts probing mnemonic processes relied on higher-order tiers of the gradient while contexts probing visual perception relied on lower tiers (Murphy et al. 2018; Murphy et al. 2019); in addition, brain regions on higher-order tiers reacted intensely to contexts with abundant semantic information while lower-tier regions were more responsive when semantic information was relatively deprived (Lanzoni et al. 2020; Wang et al. 2020). These results are consistent with our present findings that the higher- and lower-tiers of the gradient were loaded on task-conditions that entailed semantic and visuospatial processes, respectively. While using distinct task manipulations and stimuli, these studies 
provide converging evidence that the transformational workflow of information computing - from sensory-motoric to abstract-cognitive - is the most dominant factors that shapes the human brain's macroscale architecture and its functional reaction to different situations. More broadly, these data can be interpreted with an evolutionary perspective (Buckner and Krienen 2013; Krienen and Sherwood 2017): In the process of phylogenetic evolution, the cortical mantle of mammalian brain drastically expanded from the primary sensory-motoric cortex to form higher-order association cortex. The default-mode system is the evolutionarily most recent structure of mammalian nervous system and most expansive in the human brain, which endows humans the ability of abstract thinking and explains why it is more reactive to internally-oriented cognition than to externally-oriented perception/action. By contrast, the sensorimotor system is evolutionarily ancient, which explains why sensory-motor regions tend to be situated at lower tiers of the gradient and occupy a circumscribed range of cortical zone (as they are the 'starting points' of cortical expansion), unlike higher-order systems that tend to have widely distributed/fragmented configurations. More research is necessary to further clarify how different subsystems' position on the gradient contributes to different aspects of human cognition. For example, in the present study we showed that the multiple-demand system tends to be situated at middle-range tiers, which might bestow this system the capacity to mediate between high-level abstract behavioural aims and low-level perception and action (Duncan et al. 2020). There has been evidence demonstrating that the multiple-demand system can be fractionated into two subsystems, with one more connected with lower-level regions (sensory-motoric) and the other connected with higher-level regions (default-mode; Dixon et al. 2018). Such bipartite fractionation has also been observed between the default-mode system and the language system (Braga and Buckner 2017; Braga et al. 2020; Chiou et al. 2020). These recent findings suggest a promising direction for future research to investigate whether the polar extremes of cortical gradient (i.e., the default-mode and sensory-motoric systems) may function as 'attractors' or 'anchor points', which drive the bipartite split seen in multiple sub-systems in the intermediary cortical zones.

\section{References}

Andrews-Hanna JR, Reidler JS, Sepulcre J, Poulin R, Buckner RL. 2010. Functional-anatomic fractionation of the brain's default network. Neuron. 65:550-562.

Barbey AK, Koenigs M, Grafman J. 2013. Dorsolateral prefrontal contributions to human working memory. cortex. 49:1195-1205.

Blank IA, Fedorenko E. 2017. Domain-general brain regions do not track linguistic input as closely as languageselective regions. Journal of Neuroscience. 37:9999-10011.

Braga RM, Buckner RL. 2017. Parallel interdigitated distributed networks within the individual estimated by intrinsic functional connectivity. Neuron. 95:457-471. e455.

Braga RM, DiNicola LM, Becker HC, Buckner RL. 2020. Situating the left-lateralized language network in the broader organization of multiple specialized large-scale distributed networks. Journal of neurophysiology. 124:1415-1448. 
bioRxiv preprint doi: https://doi.org/10.1101/2021.1126.470178; this version posted November $26,2021$. The copyright holder for this preprint (which was not certified by peer review) is the author/funder, who has granted bioRxiv a license to display the preprint in perpetuity. It is made available under aCC-BY-NC-ND 4.0 International license.

Buckner RL, Krienen FM. 2013. The evolution of distributed association networks in the human brain. Trends in cognitive sciences. 17:648-665.

Chiou R, Humphreys GF, Jung J, Lambon Ralph MA. 2018. Controlled semantic cognition relies upon dynamic and flexible interactions between the executive 'semantic control'and hub-and-spoke 'semantic representation'systems. Cortex. 103:100-116.

Chiou R, Humphreys GF, Lambon Ralph MA. 2020. Bipartite functional fractionation within the default network supports disparate forms of internally oriented cognition. Cerebral Cortex. 30:5484-5501.

Chiou R, Lambon Ralph MA. 2016. Task-Related Dynamic Division of Labor Between Anterior Temporal and Lateral Occipital Cortices in Representing Object Size. The Journal of Neuroscience. 36:4662-4668.

Chiou R, Lambon Ralph MA. 2019. Unveiling the dynamic interplay between the hub-and spoke-components of the brain's semantic system and its impact on human behaviour. NeuroImage. 199:114-126.

Clarke A, Tyler LK. 2015. Understanding what we see: how we derive meaning from vision. Trends in cognitive sciences. 19:677-687.

Cole MW, Reynolds JR, Power JD, Repovs G, Anticevic A, Braver TS. 2013. Multi-task connectivity reveals flexible hubs for adaptive task control. Nature neuroscience. 16:1348-1355.

Davey J, Thompson HE, Hallam G, Karapanagiotidis T, Murphy C, De Caso I, Krieger-Redwood K, Bernhardt BC, Smallwood J, Jefferies E. 2016. Exploring the role of the posterior middle temporal gyrus in semantic cognition: Integration of anterior temporal lobe with executive processes. NeuroImage. 137:165-177.

Duncan J. 2010. The multiple-demand (MD) system of the primate brain: mental programs for intelligent behaviour. Trends in cognitive sciences. 14:172-179.

Duncan J, Assem M, Shashidhara S. 2020. Integrated intelligence from distributed brain activity. Trends in Cognitive Sciences. 24:838-852.

Fedorenko E. 2014. The role of domain-general cognitive control in language comprehension. Frontiers in psychology. $5: 335$.

Fedorenko E, Behr MK, Kanwisher N. 2011. Functional specificity for high-level linguistic processing in the human brain. Proceedings of the National Academy of Sciences. 108:16428-16433.

Fedorenko E, Blank IA. 2020. Broca's area is not a natural kind. Trends in cognitive sciences. 24:270-284.

Fedorenko E, Blank IA, Siegelman M, Mineroff Z. 2020. Lack of selectivity for syntax relative to word meanings throughout the language network. Cognition. 203:104348.

Fedorenko E, Duncan J, Kanwisher N. 2013. Broad domain generality in focal regions of frontal and parietal cortex. Proceedings of the National Academy of Sciences. 110:16616-16621.

Glasser MF, Coalson TS, Robinson EC, Hacker CD, Harwell J, Yacoub E, Ugurbil K, Andersson J, Beckmann CF, Jenkinson M. 2016. A multi-modal parcellation of human cerebral cortex. Nature. 536:171-178.

Hoffman P, Binney RJ, Ralph MAL. 2015. Differing contributions of inferior prefrontal and anterior temporal cortex to concrete and abstract conceptual knowledge. Cortex. 63:250-266.

Humphreys GF, Lambon Ralph MA. 2017. Mapping domain-selective and counterpointed domain-general higher cognitive functions in the lateral parietal cortex: evidence from fMRI comparisons of difficulty-varying semantic versus visuo-spatial tasks, and functional connectivity analyses. Cerebral Cortex. 27:4199-4212.

Jackson RL. 2020. The neural correlates of semantic control revisited. NeuroImage. 224:117444.

Jackson RL, Hoffman P, Pobric G, Lambon Ralph MA. 2015. The Nature and Neural Correlates of Semantic Association versus Conceptual Similarity. Cerebral cortex. 25:4319-4333. 
bioRxiv preprint doi: https://doi.org/10.1101/2021.1126.470178; this version posted November 26,2021 . The copyright holder for this preprint (which was not certified by peer review) is the author/funder, who has granted bioRxiv a license to display the preprint in perpetuity. It is made available under aCC-BY-NC-ND 4.0 International license.

Jackson RL, Rogers TT, Ralph MAL. 2021. Reverse-engineering the cortical architecture for controlled semantic cognition. Nature Human Behaviour.1-13.

Jefferies E, Thompson H, Cornelissen P, Smallwood J. 2020. The neurocognitive basis of knowledge about object identity and events: dissociations reflect opposing effects of semantic coherence and control. Philosophical Transactions of the Royal Society B. 375:20190300.

Jung J, Lambon Ralph MA. 2016. Mapping the dynamic network interactions underpinning cognition: a cTBS-fMRI study of the flexible adaptive neural system for semantics. Cerebral Cortex. 26:3580-3590.

Krienen FM, Sherwood CC. 2017. Gradients of connectivity in the cerebral cortex. Trends in cognitive sciences. 21:6163.

Kundu P, Brenowitz ND, Voon V, Worbe Y, Vértes PE, Inati SJ, Saad ZS, Bandettini PA, Bullmore ET. 2013. Integrated strategy for improving functional connectivity mapping using multiecho fMRI. Proceedings of the National Academy of Sciences. 110:16187-16192.

Kundu P, Inati SJ, Evans JW, Luh W-M, Bandettini PA. 2012. Differentiating BOLD and non-BOLD signals in fMRI time series using multi-echo EPI. Neuroimage. 60:1759-1770.

Kundu P, Voon V, Balchandani P, Lombardo MV, Poser BA, Bandettini PA. 2017. Multi-echo fMRI: a review of applications in fMRI denoising and analysis of BOLD signals. Neuroimage. 154:59-80.

Lambon Ralph MA, Jefferies E, Patterson K, Rogers TT. 2017. The neural and computational bases of semantic cognition. Nature Reviews Neuroscience. 18:42-55.

Lanzoni L, Ravasio D, Thompson H, Vatansever D, Margulies D, Smallwood J, Jefferies E. 2020. The role of default mode network in semantic cue integration. NeuroImage. 219:117019.

Margulies DS, Ghosh SS, Goulas A, Falkiewicz M, Huntenburg JM, Langs G, Bezgin G, Eickhoff SB, Castellanos FX, Petrides M. 2016. Situating the default-mode network along a principal gradient of macroscale cortical organization. Proceedings of the National Academy of Sciences. 113:12574-12579.

Margulies DS, Ovadia-Caro S, Saadon-Grosman N, Bernhardt B, Jefferies B, Smallwood J. 2020. Cortical Gradients and Their Role in Cognition. In. Reference Module in Neuroscience and Biobehavioral Psychology Elsevier.

Murphy C, Jefferies E, Rueschemeyer S-A, Sormaz M, Wang H-t, Margulies DS, Smallwood J. 2018. Distant from input: Evidence of regions within the default mode network supporting perceptually-decoupled and conceptually-guided cognition. NeuroImage. 171:393-401.

Murphy C, Wang H-T, Konu D, Lowndes R, Margulies DS, Jefferies E, Smallwood J. 2019. Modes of operation: A topographic neural gradient supporting stimulus dependent and independent cognition. NeuroImage. 186:487-496.

Noonan KA, Jefferies E, Visser M, Lambon Ralph MA. 2013. Going beyond inferior prefrontal involvement in semantic control: evidence for the additional contribution of dorsal angular gyrus and posterior middle temporal cortex. Journal of cognitive neuroscience. 25:1824-1850.

Oligschläger S, Huntenburg JM, Golchert J, Lauckner ME, Bonnen T, Margulies DS. 2017. Gradients of connectivity distance are anchored in primary cortex. Brain Structure and Function. 222:2173-2182.

Power JD, Cohen AL, Nelson SM, Wig GS, Barnes KA, Church JA, Vogel AC, Laumann TO, Miezin FM, Schlaggar BL. 2011. Functional network organization of the human brain. Neuron. 72:665-678.

Raichle ME, MacLeod AM, Snyder AZ, Powers WJ, Gusnard DA, Shulman GL. 2001. A default mode of brain function. Proceedings of the National Academy of Sciences. 98:676-682.

Rice GE, Caswell H, Moore P, Lambon Ralph MA, Hoffman P. 2018. Revealing the dynamic modulations that underpin a resilient neural network for semantic cognition: an fmri investigation in patients with anterior temporal lobe resection. Cerebral Cortex. 28:3004-3016.

Rogalski E, Cobia D, Harrison TM, Wieneke C, Thompson CK, Weintraub S, Mesulam M-M. 2011. Anatomy of language impairments in primary progressive aphasia. Journal of neuroscience. 31:3344-3350. 
bioRxiv preprint doi: https://doi.org/10.1101/2021.1126.470178; this version posted November 26, 2021. The copyright holder for this preprint (which was not certified by peer review) is the author/funder, who has granted bioRxiv a license to display the preprint in perpetuity. It is made available under aCC-BY-NC-ND 4.0 International license.

Sanchez-Panchuelo RM, Francis S, Bowtell R, Schluppeck D. 2010. Mapping human somatosensory cortex in individual subjects with 7T functional MRI. Journal of neurophysiology. 103:2544-2556.

Shashidhara S, Mitchell DJ, Erez Y, Duncan J. 2019. Progressive recruitment of the frontoparietal multiple-demand system with increased task complexity, time pressure, and reward. Journal of cognitive neuroscience. 31:1617-1630.

Shashidhara S, Spronkers FS, Erez Y. 2020. Individual-subject functional localization increases Univariate activation but not multivariate pattern discriminability in the "multiple-demand" frontoparietal network. Journal of cognitive neuroscience. 32:1348-1368.

Smallwood J, Turnbull A, Wang H-t, Ho NS, Poerio GL, Karapanagiotidis T, Konu D, Mckeown B, Zhang M, Murphy C. 2021. The neural correlates of ongoing conscious thought. Iscience.102132.

Treisman AM, Gelade G. 1980. A feature-integration theory of attention. Cogn Psychol. 12:97-136.

Uddin LQ, Yeo BT, Spreng RN. 2019. Towards a universal taxonomy of macro-scale functional human brain networks. Brain topography.1-17.

Van Heuven WJ, Mandera P, Keuleers E, Brysbaert M. 2014. SUBTLEX-UK: A new and improved word frequency database for British English. Quarterly journal of experimental psychology. 67:1176-1190.

Visser M, Jefferies E, Lambon Ralph MA. 2010. Semantic processing in the anterior temporal lobes: a meta-analysis of the functional neuroimaging literature. J Cogn Neurosci. 22:1083-1094.

Wandell BA, Dumoulin SO, Brewer AA. 2007. Visual field maps in human cortex. Neuron. 56:366-383.

Wang X, Gao Z, Smallwood J, Jefferies E. 2021. Both default and multiple-demand regions represent semantic goal information. Journal of Neuroscience.

Wang X, Margulies DS, Smallwood J, Jefferies E. 2020. A gradient from long-term memory to novel cognition: Transitions through default mode and executive cortex. NeuroImage. 220:117074.

Wehbe L, Blank IA, Shain C, Futrell R, Levy R, von der Malsburg T, Smith N, Gibson E, Fedorenko E. 2021. Incremental language comprehension difficulty predicts activity in the language network but not the multiple demand network. Cerebral Cortex. 31:4006-4023.

Wig GS, Laumann TO, Petersen SE. 2014. An approach for parcellating human cortical areas using resting-state correlations. Neuroimage. 93:276-291.

Wilson SM, Bautista A, McCarron A. 2018. Convergence of spoken and written language processing in the superior temporal sulcus. Neuroimage. 171:62-74.

Woolgar A, Duncan J, Manes F, Fedorenko E. 2018. Fluid intelligence is supported by the multiple-demand system not the language system. Nature Human Behaviour. 2:200-204.

Yeo BTT, Krienen FM, Sepulcre J, Sabuncu MR, Lashkari D, Hollinshead M, Roffman JL, Smoller JW, Zöllei L, Polimeni JR, Fischl B, Liu H, Buckner RL. 2011. The organization of the human cerebral cortex estimated by intrinsic functional connectivity. Journal of Neurophysiology. 106:1125-1165. 


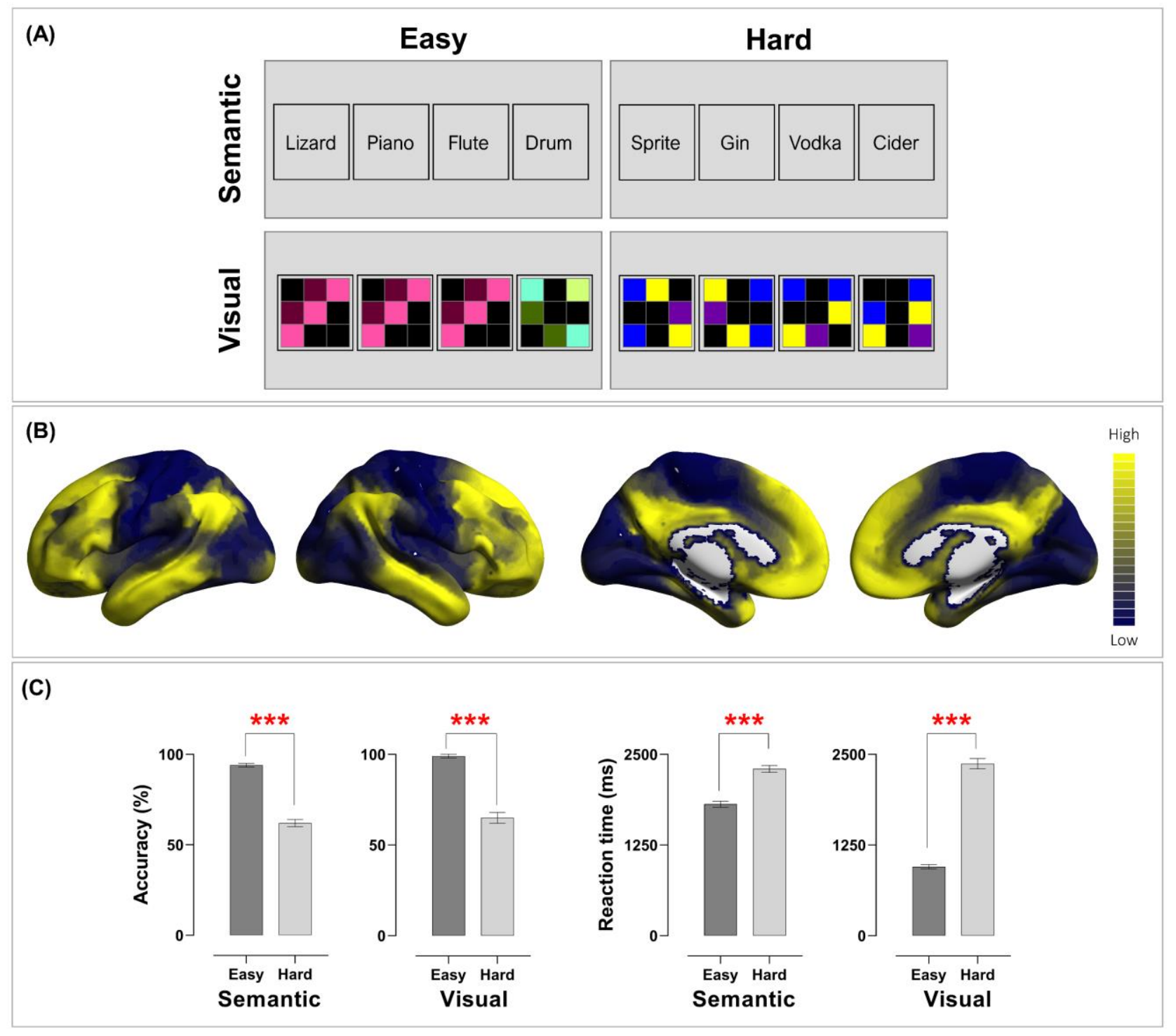

Figure 1. (A) Example stimuli of the four conditions. In this $2 \times 2$ factorial design, the type of cognitive operation (Semantic vs. Visuospatial) and the extent of cognitive effort needed to identify an oddball (Easy vs. Hard) were independently manipulated. (B) The principal gradient that regulates the brain's connectivity pattern, identified by Margulies et al. (2016). Brain regions that fall on the topmost tier tend to belong to the default-mode and language-specific systems, whereas those fall in the lowest tier belong to the sensory-motoric system. (C) In both accuracy and reaction time, the difficulty effect was robustly found in both Semantic and Visuospatial conditions. 
(A)
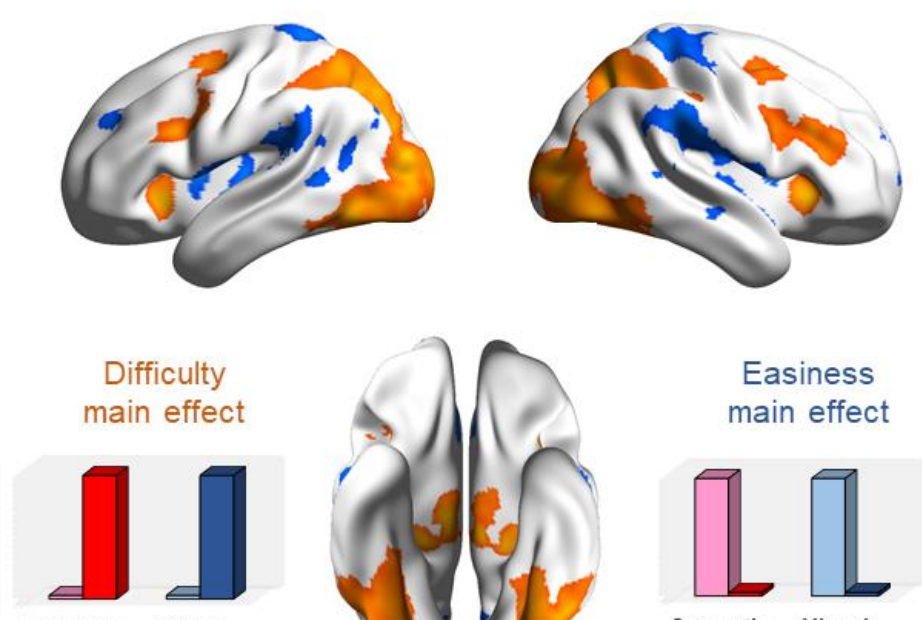

Semantic Visual

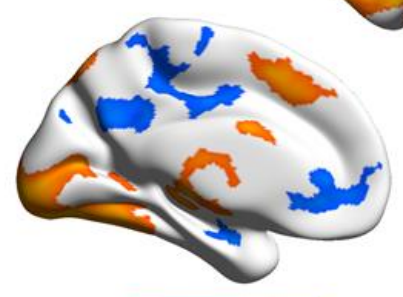

$-10$
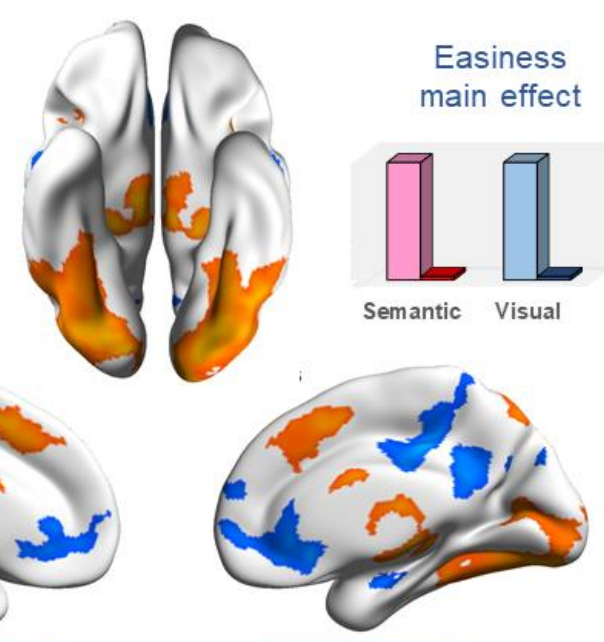

3.5

10
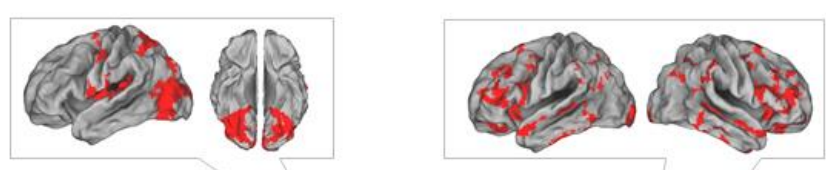

(B)
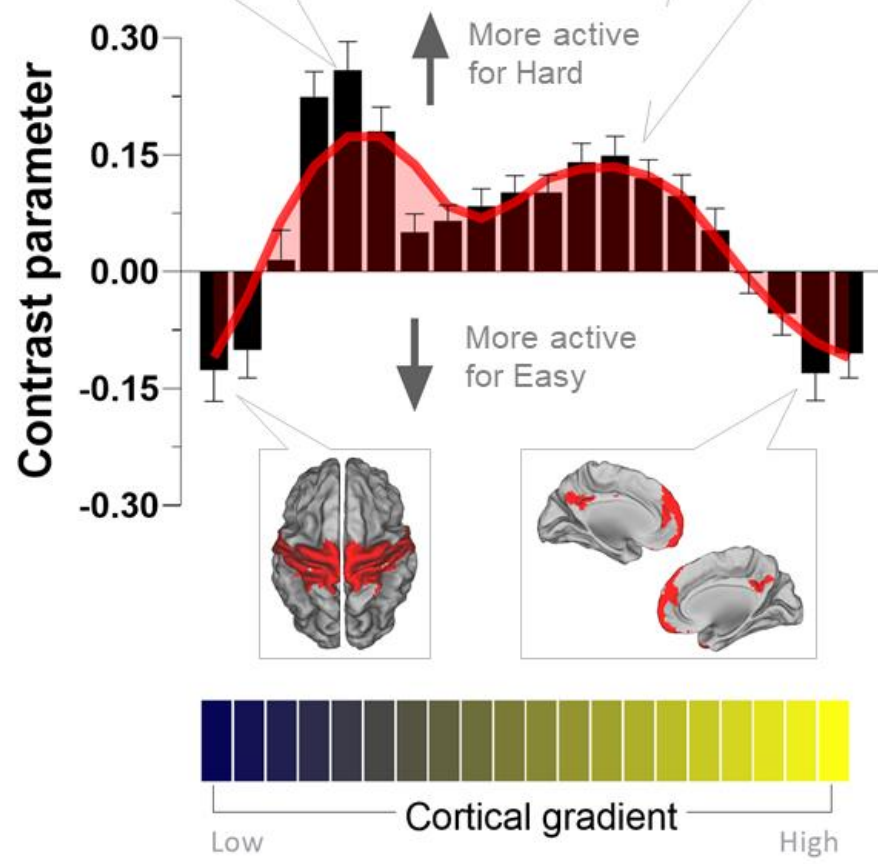

Figure 2. (A) The GLM-contrast results of Easy vs. Hard: Warm colour rendered on the brain indicates the main effect of difficulty (Semantic-Hard + Visuospatial-Hard > Semantic-Easy + Visuospatial-Easy); cold colour indicated the reverse contrast (the main effect of easiness). (B) The results of GLM-contrast were projected onto the principal gradient of the human brain. 
(A)
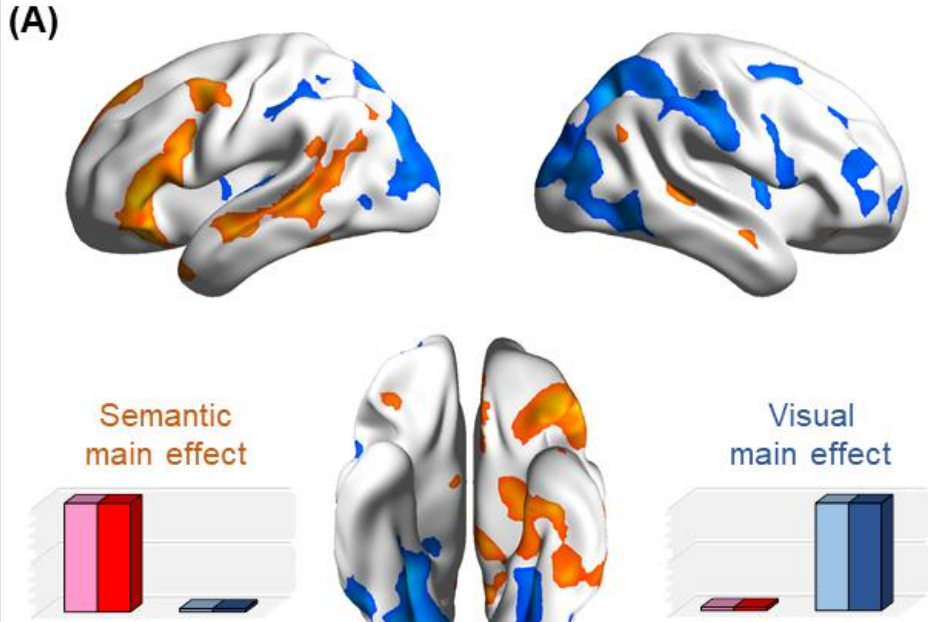

Semantic Visual
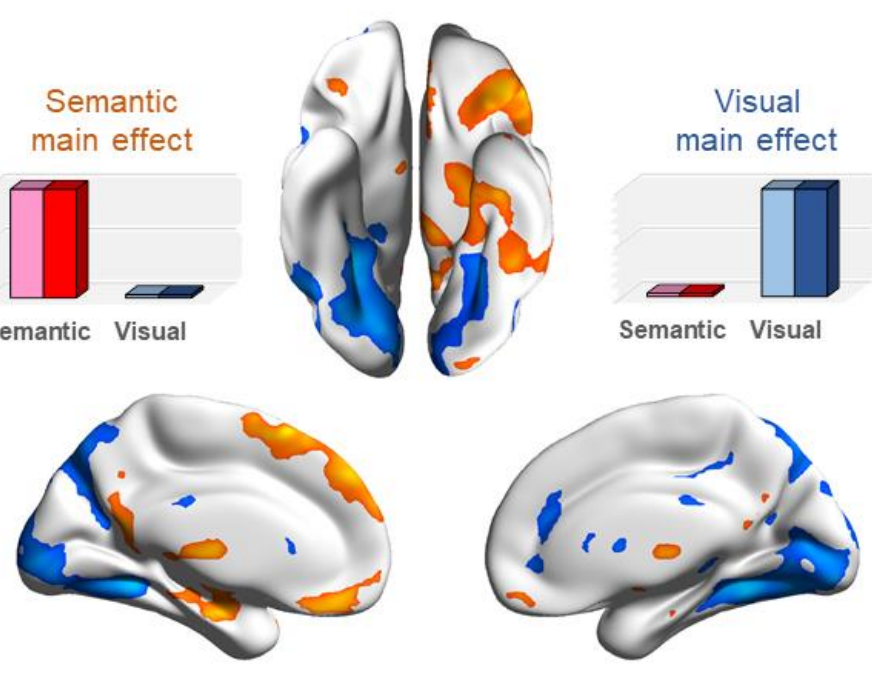

$-9$ $-3.5$

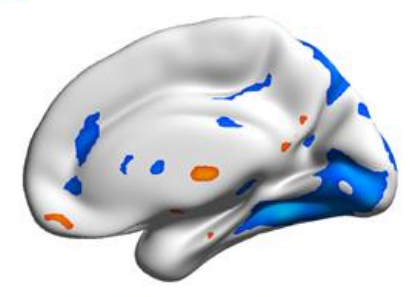

3.5

8

Figure 3. (A) The GLM-contrast results of Semantic vs. Visuospatial: Warm colour rendered on the brain indicates the main effect of semantic processing (Semantic-Easy + Semantic-Hard > Visuospatial-Easy + Visuospatial-Hard); cold colour indicated the reverse contrast (the main effect of visuospatial processing). (B) The results of GLM-contrast were projected onto the principal gradient of the human brain. 


\section{(A)}

\section{Regions sensitive to cognitive control}

Enhanced demand of semantic control (SemHard > SemEasy) Enhanced demand of visuospatial control (VisHard > VisEasy) Conjunction of semantic control AND visuospatial control

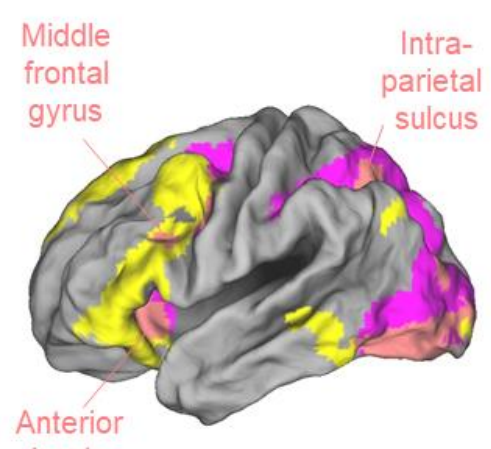

insula

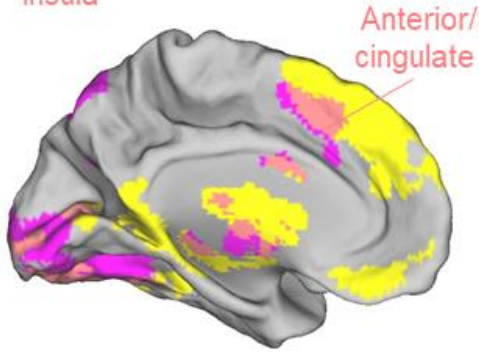

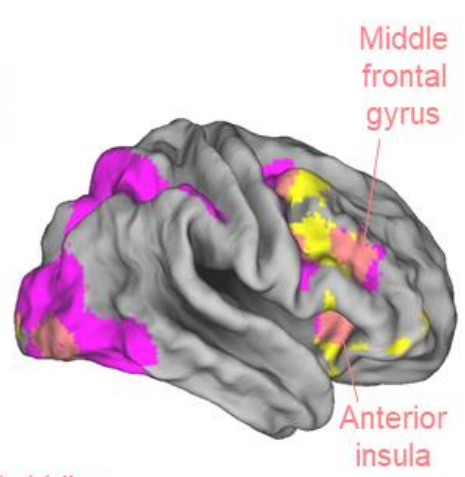

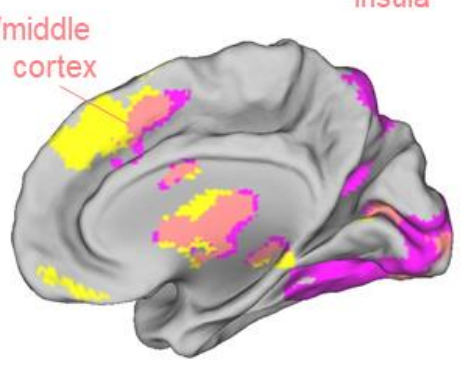

Regions preferring semantic cognition to perceptual stimuli

Preference for deeper semantics (SemHard > SemEasy) Aversion to difficult perceptual processes (VisEasy > VisHard) Conjunction of the two (more semantics AND less perception)

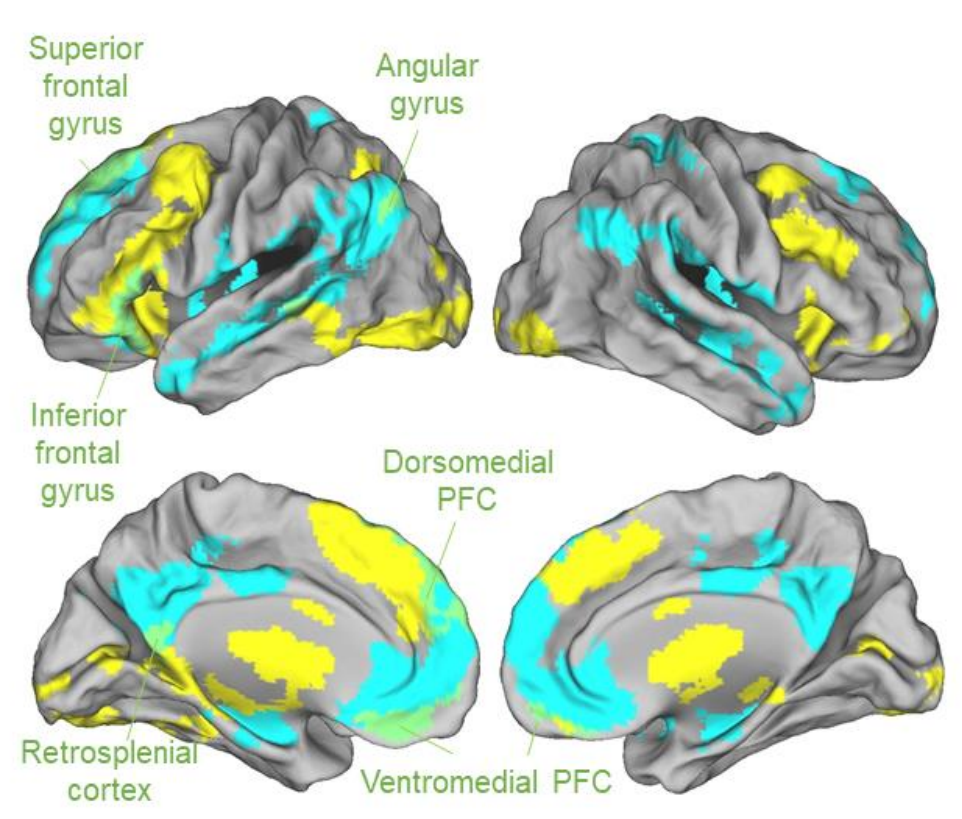

Figure 4. Yellow rendered on the brain are identical in (A) and (B); it indicates the brain regions more active for the 'Semantic-Hard > Semantic-Easy' contrast. By comparing (A) and (B), it is evident that the yellow 'semantic-control' clusters are partially overlapped with the contrast for heightened demand for visuospatial control (magenta clusters in A); however, the yellow clusters are also partially overlapped with the reverse contrast (cyan clusters in B: reduced demand for visuospatial control or higher degree of automaticity - i.e., regions more active for the behaviourally least demanding Visuospatial-Easy condition). In multiple parts of the brain, the 'semantic-control' clusters were situated in the intermediary zones between magenta and cyan. 


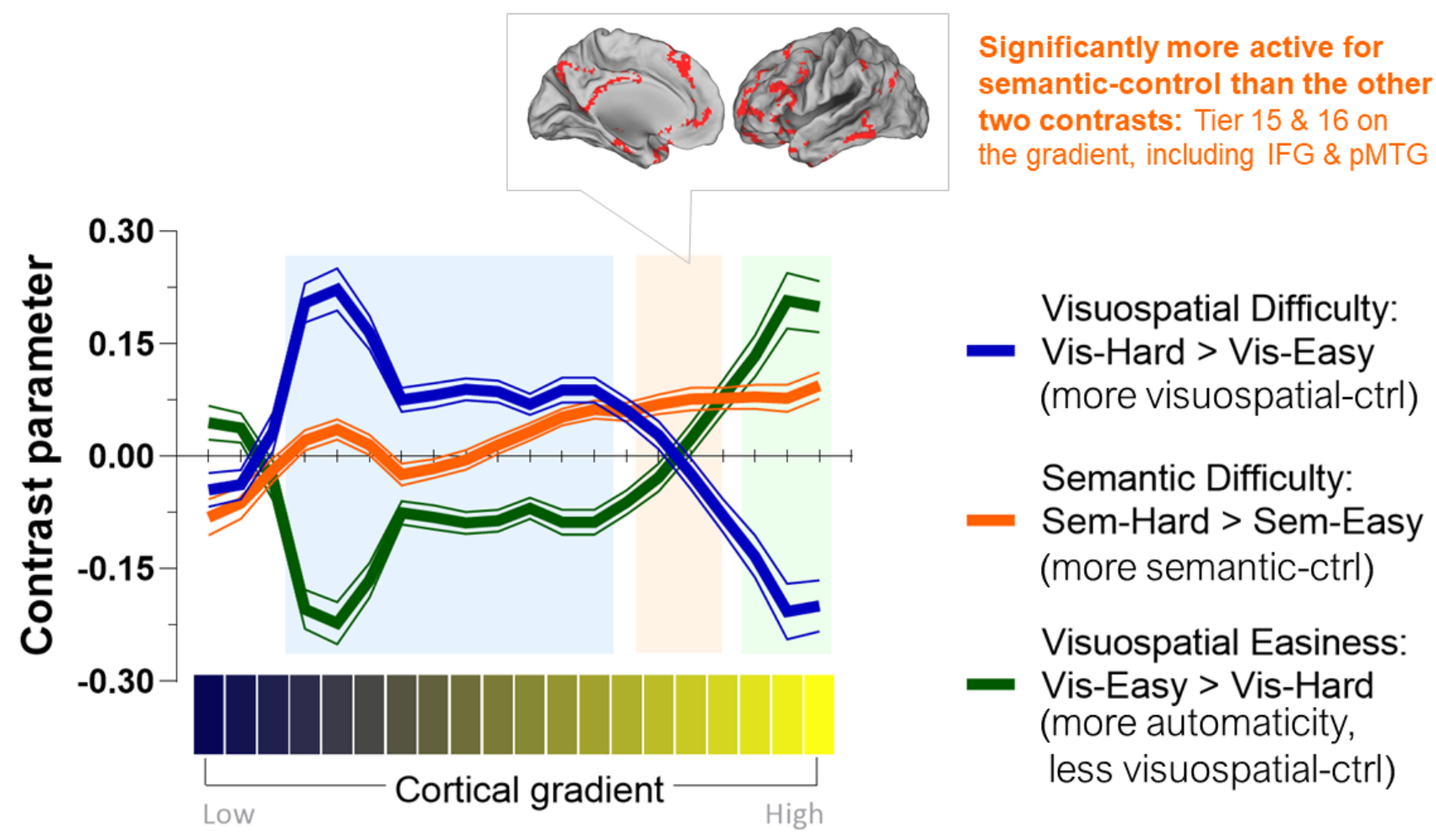

Figure 5. When the three contrasts were projected onto the principal gradient of Margulies et al. (2016), the 'Semantic-Difficulty' effect was most pronounced in intermediary tiers (shaded with orange; Tier 15 and 16) and showed significantly greater contrast parameter than the other two effects (Visuospatial-Difficulty and Easiness). These intermediary tiers were situated between low-to-middle tiers (shaded with blue; where in regions were most active for difficult visuospatial operation) and topmost tiers (shaded with green; wherein regions were most active for the behaviourally least demanding condition in which mind-wandering was more likely to arise). The size of standard errors along the gradient are indicated by the upper and lower thin lines flanking each trajectory. 
bioRxiv preprint doi: https://doi. org/10.1101/2021.11.26.470178; this version posted November 26, 2021. The copyright holder for this

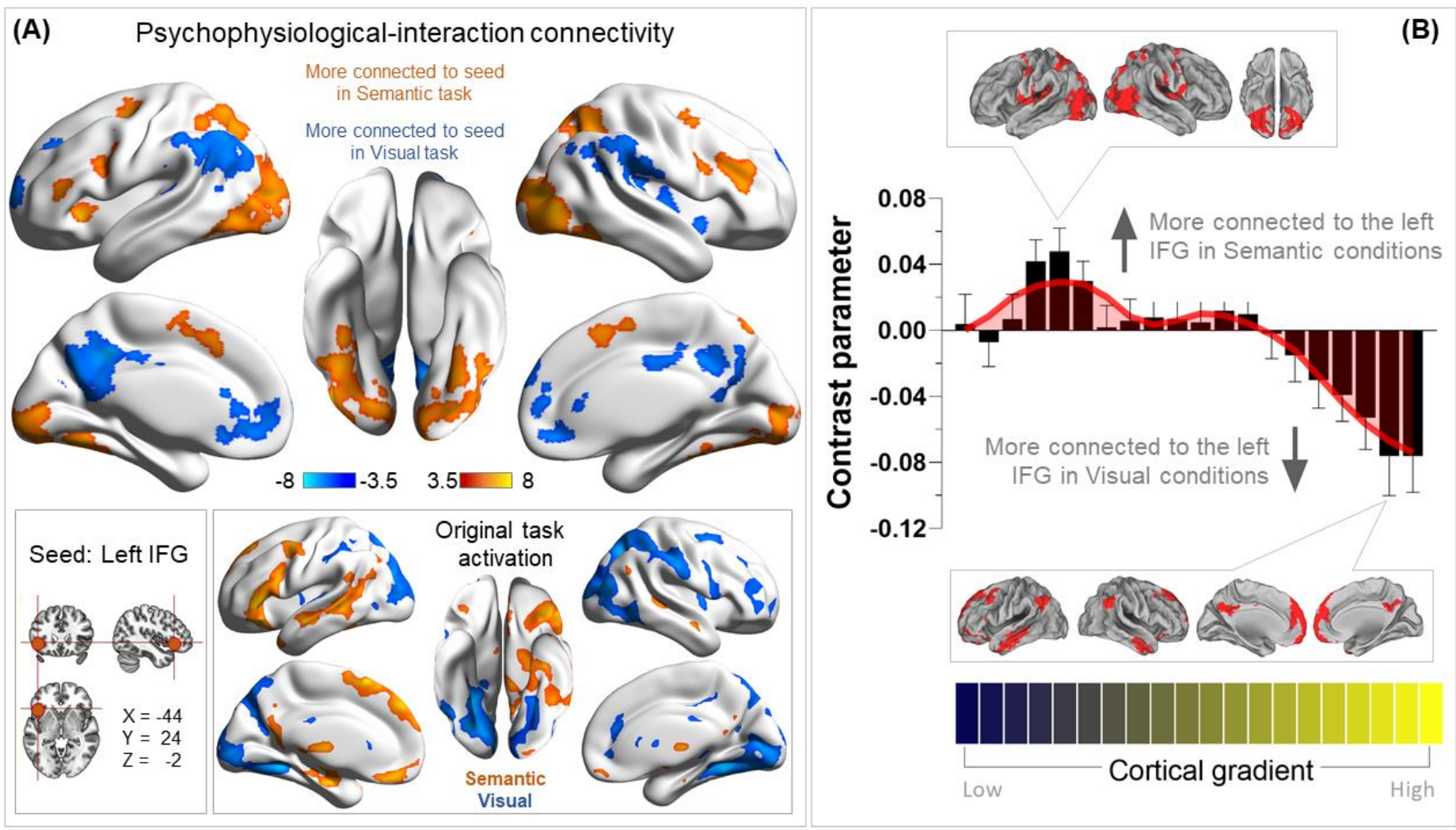

Figure 6. (A) The PPI results of contextually dependent connectivity to the seed region of left IFG, comparing how the connectivity pattern altered between the Semantic and Visuospatial conditions. (B) The results of PPI were projected onto the principal gradient of the human brain. 
bioRxiv preprint doi: https://doi org/10.1101/2021.1126.470178; this version posted November 26, 2021. The copyright holder for this preprint (which was not certified by peer review) is the author/funder, who has granted bioRxiv a license to display the preprint in perpetuity. It is made available under aCC-BY-NC-ND 4.0 International license.

(A)

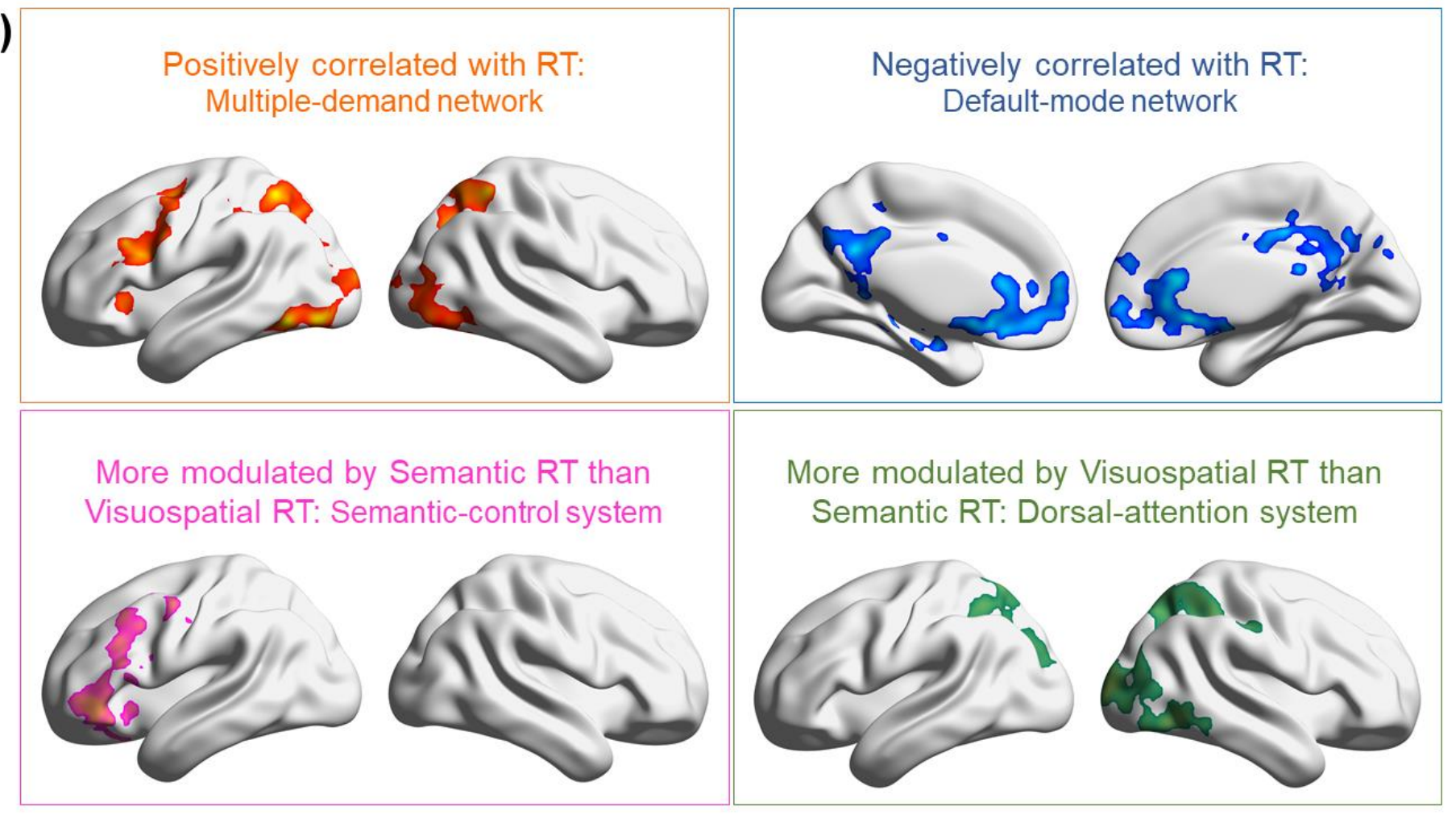

(B)

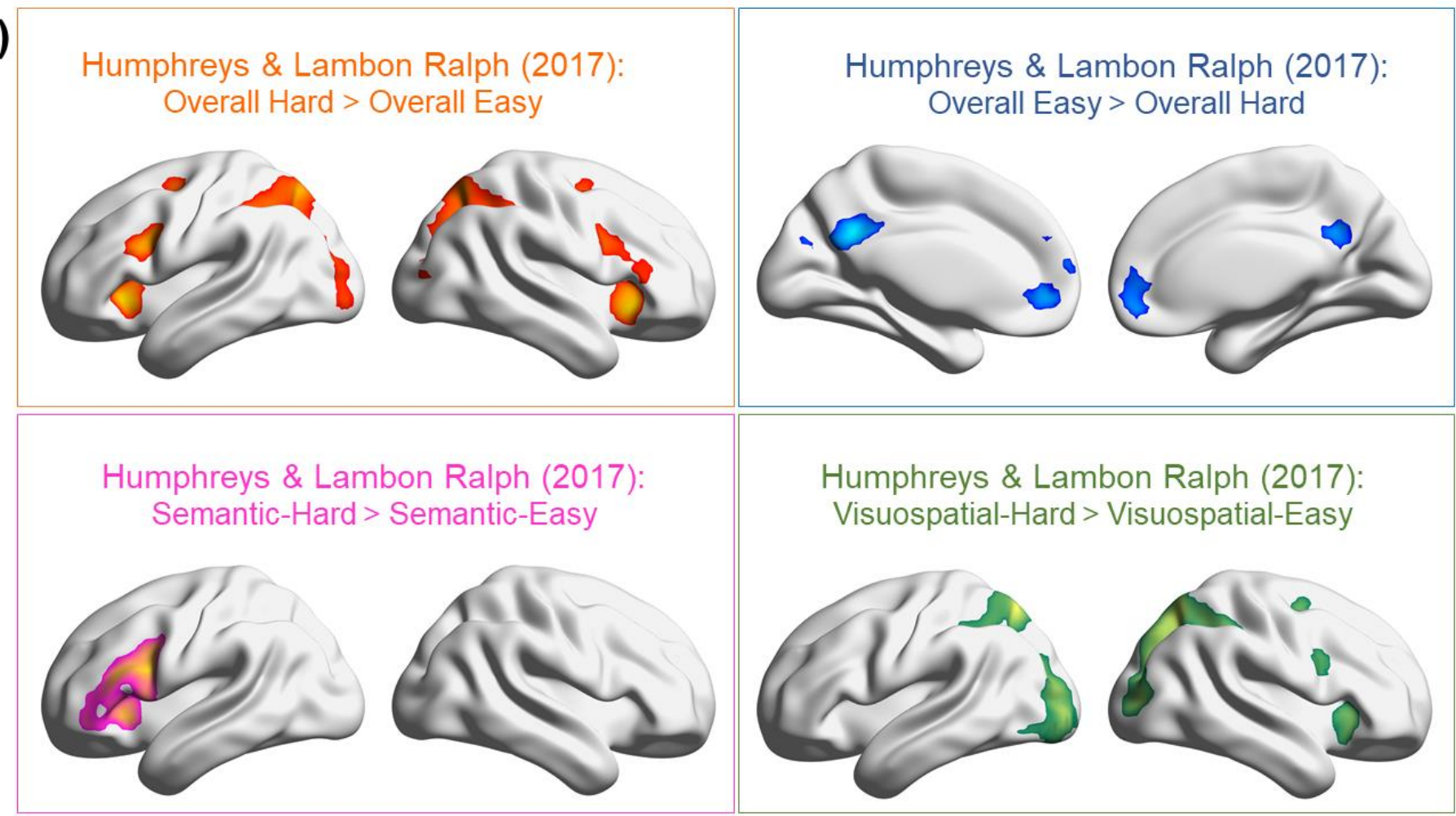

Figure 7. (A) Parametric modulation analysis of reaction times revealed four aspects of the brain's control-related machineries: domain-general positive correlation with RTs (multi-demand network), domain-general negative correlation with RTs (default-mode network), semantic control (the left IFG and adjoining regions), and visuospatial control (dorsal-attention system). (B) A strikingly consistent pattern of brain activation was replicated using the independent dataset of Humphreys and Lambon Ralph (2017). 